\title{
A suppressor of axillary meristem maturation promotes longevity in flowering plants
}

\author{
Omid Karami', Arezoo Rahimi', Majid Khan ${ }^{1,5}$, Marian Bemer ${ }^{2}$, Rashmi R. Hazarika ${ }^{3}{ }^{3}$, Patrick Mak ${ }^{1,6}$, \\ Monique Compier ${ }^{1,7}$, Vera van Noort $\mathbb{1}^{3,4}$ and Remko Offringa ${ }^{1}{ }^{1 凶}$
}

\begin{abstract}
Post-embryonic development and longevity of flowering plants are, for a large part, determined by the activity and maturation state of stem cell niches formed in the axils of leaves, the so-called axillary meristems (AMs) ${ }^{1,2}$. The genes that are associated with AM maturation and underlie the differences between monocarpic (reproduce once and die) annual and the longer-lived polycarpic (reproduce more than once) perennial plants are still largely unknown. Here we identify a new role for the Arabidopsis AT-HOOK MOTIF NUCLEAR LOCALIZED 15 (AHL15) gene as a suppressor of AM maturation. Loss of AHL15 function accelerates AM maturation, whereas ectopic expression of AHL15 suppresses AM maturation and promotes longevity in monocarpic Arabidopsis and tobacco. Accordingly, in Arabidopsis grown under longevitypromoting short-day conditions, or in polycarpic Arabidopsis lyrata, expression of AHL15 is upregulated in AMs. Together, our results indicate that $A H L 15$ and other $A H L$ clade-A genes play an important role, directly downstream of flowering genes (SOC1, FUL) and upstream of the flowering-promoting hormone gibberellic acid, in suppressing AM maturation and extending the plant's lifespan.
\end{abstract}

Plant architecture and longevity are dependent on the activity of stem cell groups called meristems. The primary shoot and root apical meristem of a plant are established during early embryogenesis and give rise, respectively, to the shoot and the root system during post-embryonic development. In flowering plants, post-embryonic shoot development begins with a vegetative phase during which the primary shoot apical meristem (SAM) produces morphogenetic units called phytomers consisting of a stem (internode) subtending a node with a leaf and a secondary or axillary meristem (AM) located in the axil of the leaf ${ }^{1,2}$. Both the SAM and these AMs undergo a maturation process. Like the SAM, young immature AMs are vegetative and, when activated, they produce leaves whereas in plant species such as Arabidopsis thaliana (Arabidopsis) partially matured AMs produce a few cauline leaves before they fully mature into inflorescence meristems and start developing phytomers comprising a stem subtending one or more flowers ${ }^{2,3}$.

The maintenance of vegetative development after flowering is an important determinant of plant longevity and life history. Monocarpic plants, such as the annuals Arabidopsis and Nicotiana tabacum (tobacco), complete their life cycle in a single growing season. The AMs that are established during the vegetative phase initially produce leaves. Following floral transition, however, all AMs rapidly convert into inflorecence meristems producing secondary and tertiary inflorescences with bracts and flowers, thus maximizing offspring production before the plant's life ends with senescence and death. The number of leaves and bracts produced by an AM is thus a measure of its maturation state following activation. By contrast, many other flowering plant species are polycarpic perennials, such as the close Arabidopsis relative A. lyrata. Under permissive growth conditions, these can live and flower for more than two growing seasons. Because some AMs are maintained in the vegetative state, this allows polycarpic plants to produce new shoots after seed set and the subsequent activation of these AMs by appropriate growth conditions before the start of the next growing season ${ }^{4,5}$. Despite considerable interest in the molecular basis of plant life history, the proposed molecular mechanisms determining the difference in loss or maintenance of vegetative development after flowering between, respectively, monocarpic and polycarpic plants are still largely based on our extensive knowledge on the control of flowering in Arabidopsis and closely related species. From these studies, the MADS box proteins SUPPRESSOR OF OVEREXPRESSION OF CONSTANS 1 (SOC1) and FRUITFULL (FUL) have been identified as key promotors of flowering and monocarpic growth, and FLOWERING LOCUS C (FLC) as their upstream coldsensitive inhibitor ${ }^{5-7}$. However, the factors that maintain vegetative development after flowering, and thereby allow polycarpic growth, remain elusive.

Here we present evidence that the Arabidopsis gene AT-HOOK MOTIF NUCLEAR LOCALIZED 15 (AHL15) plays an important role in the control of AM maturation and extending the plant's lifespan. This gene forms a clade (clade A) with 14 other $A H L$ genes in Arabidopsis that encode nuclear proteins containing a single $\mathrm{N}$-terminal DNA-binding AT-hook motif and a C-terminal plants and prokaryotes conserved (PPC) domain (Supplementary Fig. 1a). The PPC domain was previously shown to contribute to physical interaction with other AHL or nuclear proteins ${ }^{8}$. AHL15 homologues have been implicated in several aspects of plant growth and development in Arabidopsis, including hypocotyl growth and leaf senescence ${ }^{8-10}$, flower development ${ }^{11}$ and flowering time $\mathrm{e}^{10,12}$.

In contrast to other $a h l$ mutants ${ }^{10}$, ahl15 loss-of-function mutant plants (Supplementary Fig. 1b-e) flowered at the same time and developed the same number of rosette leaves before flowering as wild-type plants, under both short-day (SD) and long-day (LD) conditions (Extended Data Fig. 1d,e). After bolting, however, AMs located in the axils of rosette leaves (rosette AMs) of ahl15 mutant

'Plant Developmental Genetics, Institute of Biology Leiden, Leiden University, Leiden, the Netherlands. ${ }^{2}$ Laboratory of Molecular Biology and B.U. Bioscience, Wageningen University and Research, Wageningen, the Netherlands. ${ }^{3}$ KU Leuven, Centre of Microbial and Plant Genetics, Leuven, Belgium. ${ }^{4}$ Bioinformatics and Genomics, Institute of Biology Leiden, Leiden University, Leiden, the Netherlands. ${ }^{5}$ Present address: Institute of Biotechnology and Genetic Engineering, University of Agriculture Peshawar, Peshawar, Pakistan. ${ }^{6}$ Present address: Sanquin Plasma Products BV, Department of Product Development, Amsterdam, the Netherlands. ${ }^{7}$ Present address: Rijk Zwaan, De Lier, the Netherlands. ${ }^{凶}$ e-mail: r.offringa@biology.leidenuniv.nl 
a

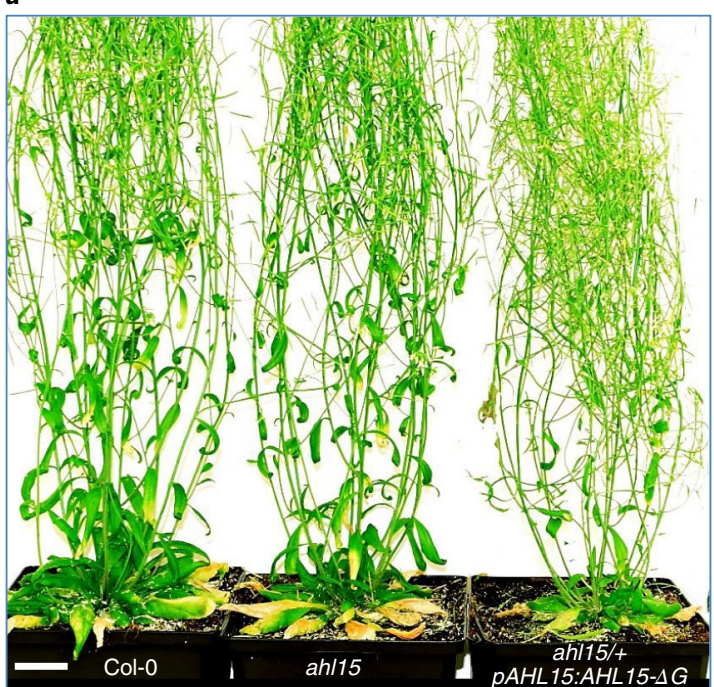

b

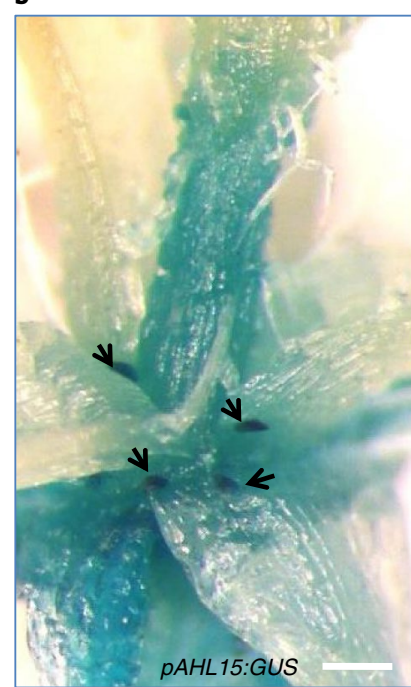

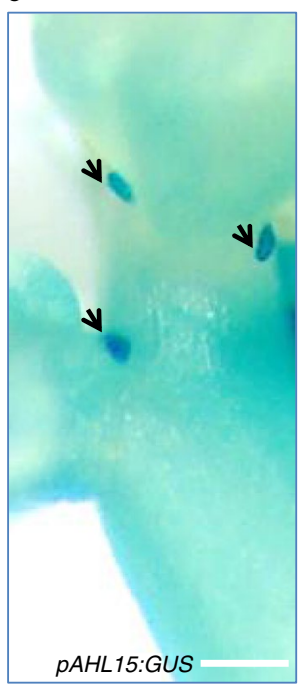

d

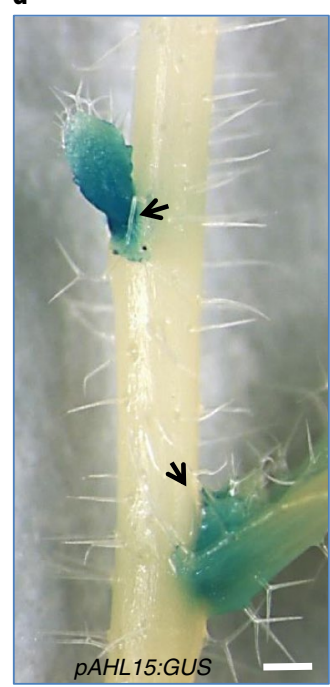

e

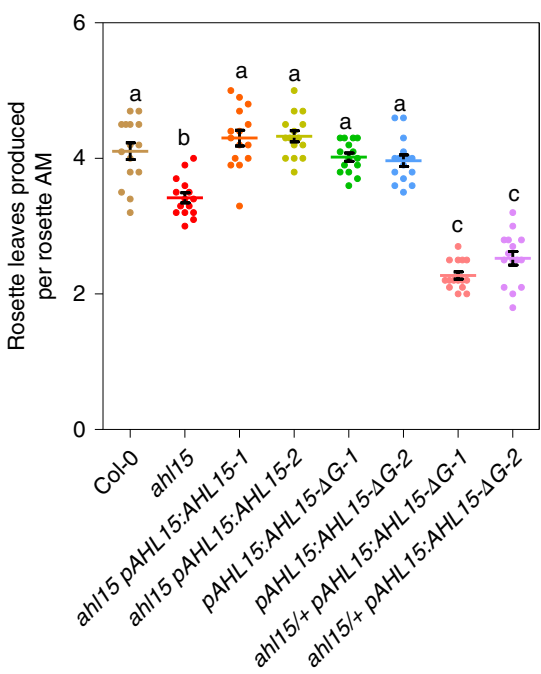

f

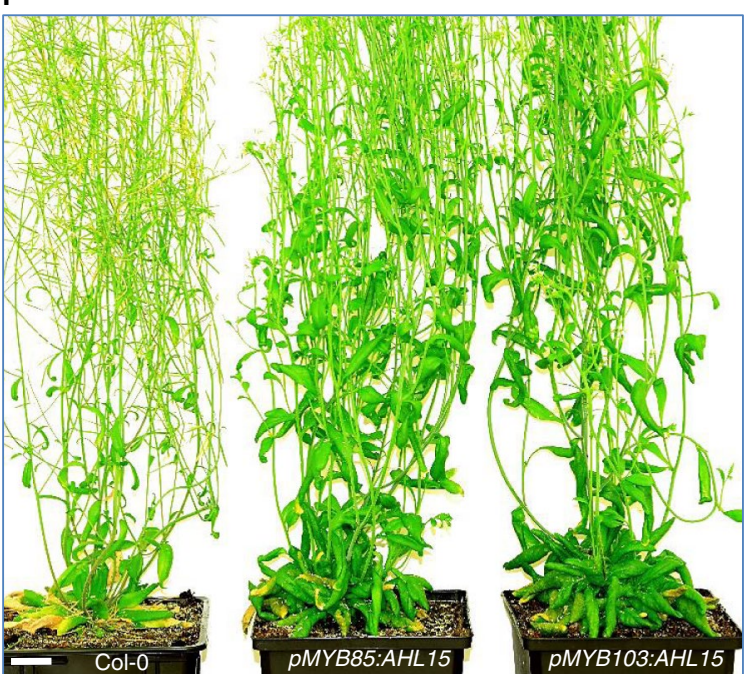

g

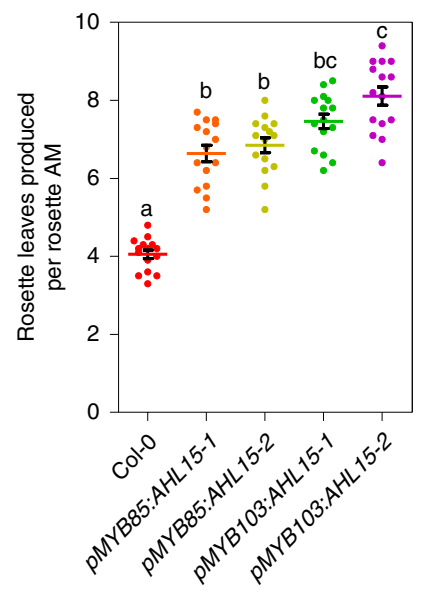

Fig. 1 | AHL15 represses AM maturation in Arabidopsis. a, Shoot phenotypes of 50-day-old flowering wild-type (left), ah/15 (middle) and ah/15/+ pAHL15:AHL15- $\Delta$ G mutant (right) plants. b-d, pAHL15:GUS expression in rosette AMs (arrowheads in b), in aerial AMs located on a young inflorescence stem (arrowheads in $\mathbf{c}$ ) and in activated axillary buds on an inflorescence stem (arrowheads in d) of a flowering plant. e, Rosette leaves produced per rosette AM in 50-day-old wild-type, ah/15, ah/15 pAHL15:AHL15, pAHL15:AHL15- $\mathrm{G}$ and ah/15/+ pAHL15:AHL15- $\Delta$ G plants. f, Shoot phenotype of a 60-dayold flowering wild-type (left), pMYB85:AHL15 (middle) and pMYB103:AHL15 (right) plant. g, Rosette leaves produced per rosette AM in 60-day-old wildtype, pMYB85:AHL15 and pMYB103:AHL15 plants. Coloured dots in e,g indicate the number of rosette leaves per plant ( $n=15$ biologically independent plants) per line, horizontal lines indicate the mean and error bars indicate s.e.m. e,g, Different letters indicate statistically significant differences $(P<0.01)$ as determined by one-way analysis of variance with Tukey's honest significant difference post hoc test. $P$ values are provided in Supplementary Tables 3 and 4. Plants were grown under LD conditions. Scale bars, $2 \mathrm{~cm}$ in $\mathbf{a}, \mathbf{f}$ and $1 \mathrm{~mm}$ in $\mathbf{b}, \mathbf{c}$. Leaf production per rosette AM in e,g was determined for two independent transgenic lines (1 and 2). For $\mathbf{a}, \mathbf{f}$, similar results were obtained from three independent experiments. For $\mathbf{b}$-d, similar results were obtained from two independent experiments.

plants produced fewer additional rosette leaves compared to those in wild-type plants (Fig. 1a,e and Extended Data Fig. 2a-c). More detailed analysis showed that this reduction in additional rosette leaf production in ahl15 plants was caused neither by delayed outgrowth of rosette AMs into axillary buds nor by early floral transition, but rather by a reduction in the vegetative activity of these buds (Extended Data Fig. 3a,b). Following floral transition, ahl15 rosette AMs produced the same number of cauline leaves (Extended Data Fig. 2d) and flowers (Supplementary Fig. 2a) as the wild type (Col-0). However, the cauline branches produced by aerial AMs on ahl15 inflorescences developed fewer cauline leaves (Extended Data Fig. 2e) and flowers/fruits (Supplementary Fig. 2b) compared to those produced by wild-type inflorescences, resulting in a significant reduction in the total number of cauline leaves and flowers on ahl15 inflorescences. Introduction of the pAHL15:AHL15 genomic clone into the ahl15 mutant background restored both the rosette and cauline leaf, as well as flower and fruit numbers to wild-type levels (Fig. 1e, Extended Data Fig. 2a-e and Supplementary Fig. 2b), confirming that the phenotypes resulted from ahl15 loss-of-function. $\beta$-Glucuronidase (GUS) staining of plants carrying a $p A H L 15: G U S$ promoter-reporter fusion showed that AHL15 is expressed in AMs (Fig. 1b,c) and young axillary buds (Fig. 1d). Together these results suggested a novel role for AHL15 in controlling AM maturation. 
AHL proteins interact with each other through their PPC domain, and with other non-AHL proteins through a conserved six-amino acid (GRFEIL) region in the PPC domain. Expression of an AHL protein without the GRFEIL region leads to a dominant negative effect, because it generates a non-functional complex that is unable to modulate transcription ${ }^{8}$. Expression of a deletion version of AHL15 lacking the GRFEIL region under control of the AHL15 promoter ( $p A H L 15: A H L 15-\Delta G)$ in the wild-type background $(n=20)$ resulted in fertile plants (Extended Data Fig. 1a,b) showing normal AM maturation (Fig. 1e and Extended Data Fig. 2). In the heterozygous ahl15 loss-of-function background, however, pAHL15:AHL15- $\triangle G$ expression induced early flowering (Extended Data Figs. 1d,e and $3 b$ ), resulting in a strong reduction in rosette and cauline leaf production by AMs (Fig. 1a,e and Extended Data Figs. 2a-f and 3a). Homozygous ahl15 pAHL15:AHL15- $\Delta$ G progeny were not obtained, and the presence of defective seeds in siliques of ahl15/+ pAHL15:AHL15- $\Delta$ G plants suggests that this genetic combination is embryo lethal (Extended Data Fig. 1b,c). The much stronger phenotypes observed for ahl15/+ pAHL15:AHL15- $\Delta G$ plants are in line with the dominant negative effect of AHL15- $\Delta \mathrm{G}$ expression, overcoming functional redundancy among Arabidopsis clade-A $A H L$ family members ${ }^{8-10}$.

Based on the observation that the flowering time and number of rosette leaves before bolting were the same for both wildtype and ahl15 loss-of-function plants, but not for ahl15/+ pAHL15:AHL15- $\Delta G$ plants, we speculated that other $A H L$ cladeA family members are more active in the SAM, whereas AHL15 acts more strongly on AM maturation. To test this, we overexpressed a fusion protein between AHL15 and the rat glucocorticoid receptor under control of the constitutive cauliflower mosaic virus $35 S$ promoter ( $p 35 S: A H L 15-G R$ ). This rendered the nuclear import, and thereby the activity of the ectopically expressed AHL15-GR fusion, inducible by dexamethasone (DEX). Untreated p35S:AHL15-GR plants showed a wild-type phenotype (Extended Data Fig. 4b,c) but, after spraying flowering p35S:AHL15-GR plants with DEX, rosette AMs produced significantly more rosette and cauline leaves (Extended Data Fig. $4 \mathrm{a}-\mathrm{c}$ ). Interestingly, spraying $p 35 S$ :AHL15-GR plants before flowering also significantly delayed their floral transition (Extended Data Fig. 4d), indicating that ectopically expressed AHL15 can also suppress maturation of the SAM. In turn, overexpression of the Arabidopsis AHL family members AHL19, AHL20, AHL27 and AHL29, as well as the putative AHL15 orthologues from Brassica oleracea and Medicago truncatula in Arabidopsis, resulted in morphological changes similar to those observed for p35S:AHL15-GR plants after DEX treatment. The overexpression plants produced more rosette and cauline leaves during flowering (Extended Data Fig. 5), supporting the idea that there is functional redundancy among $A H L$ clade-A family members and that the ability to control either SAM or AM maturation is dependent on the spatio-temporal expression of the corresponding genes.

In contrast to the observed growth arrest and death of 2-monthold Arabidopsis plants grown under LD conditions (Fig. 2d), 4-month-old SD Arabidopsis plants continued to grow after the first cycle of flowering because aerial AMs on the last-formed lateral branches produced new rosette leaves (Fig. 2a and Extended Data Fig. 6). However, SD ahl15 mutant plants did not show this renewed vegetative growth and died, whereas ahl15 pAHL15:AHL15 plants grew like the wild type under these conditions (Fig. 2a and Extended Data Fig. 6). GUS staining of $p A H L 15$ :GUS plants revealed that AHL15 expression was strongly enhanced in young lateral inflorescences, axils of cauline leaves and rosette branches under SD conditions compared to LD conditions (Fig. 2b,c and Extended Data Fig. 7), indicating that AHL15 expression is day length sensitive and confirming the important role of this gene in extending the lifespan of Arabidopsis under SD conditions.
Growing Arabidopsis plants under SD conditions significantly delays flowering ${ }^{13}$, and this might thus indirectly enhance the repressive effect of AHL15 on AM maturation and extend the lifespan. To assess AHL15 function independently of day length and flowering time, we expressed AHL15 under the control of promoters pMYB85 or pMYB103, which are highly active in Arabidopsis rosette nodes and aerial axillary buds (Extended Data Fig. 8a) ${ }^{14}$. Under LD conditions, pMYB85:AHL15 and pMYB103:AHL15 plants flowered at the same time as wild-type plants, but their AMs produced significantly more rosette and cauline leaves compared to those in wild-type plants (Fig. 1f,g and Extended Data Fig. 8b,c). Moreover, after flowering and seed set, when wild-type plants senesced and died, pMYB85:AHL15 and pMYB103:AHL15 rosette and aerial AMs produced new rosette leaves, which allowed these plants to continue to grow and generate new flowers and seeds (Fig. 2d,e). In addition, senesced p35S:AHL15-GR plants carrying fully ripened siliques began new aerial vegetative development on lateral secondary inflorescences after DEX treatment, and ultimately produced new inflorescences from the resulting rosettes (Extended Data Fig. 9a). Interestingly, the development of vegetative shoots from AMs formed on rosette and aerial nodes after reproduction also contributes to the polycarpic growth habit of Arabis alpina and Cardamine flexuosa plants ${ }^{15,16}$. Our results indicate that increased expression of AHL15 in the late stages of development promotes longevity by inducing a polycarpic-like growth habit in Arabidopsis, with an important difference that AMs remaining vegetative do not show dormancy.

To determine whether heterologous AHL15 expression could induce similar developmental changes in a monocarpic plant species from a different family, we introduced the 35S:AHL15-GR construct into tobacco. Wild-type and p35S:AHL15-GR tobacco plants were allowed to grow and set seeds in the absence of DEX treatment. After seed harvesting, all leaves and side branches were removed and the bare lower parts of the primary stems were either mock or DEX treated. Whereas stems of wild-type and mock-treated p35S:AHL15-GR plants did not show any growth, AMs on DEXtreated $p 35 S: A H L 15-G R$ tobacco stems resumed vegetative growth, eventually leading to a second cycle of flowering and seed set (Fig. 2f). Continued DEX treatment after each subsequent cycle of seed harvesting efficiently induced vegetative growth and subsequent flowering and seed set, allowing $p 35 S$ :AHL15-GR tobacco plants to survive at least 3 years (Extended Data Fig. 9b). This result confirmed the conclusion from previous overexpression experiments (Fig. 2d,e and Extended Data Figs. 4 and 9a) that enhanced AHL15 expression facilitates polycarpic-like growth by maintaining some AMs in the vegetative state after flowering.

Previously, loss of function of both SOC1 and FUL in Arabidopsis was reported to suppress AM maturation, resulting in polycarpiclike growth ${ }^{6}$. We found that the aerial rosette formation normally observed in the soc1 ful double mutant was significantly reduced in soc1 ful ahl15 triple-mutant plants (Fig. 3a,b). Moreover, polycarpic features of the soc1 ful double mutant were lost in the ahl15 mutant background because soc1 ful ahl15 plants senesced and died following seed set, just like wild-type Arabidopsis. Expression analysis by quantitative PCR (qPCR) with reverse transcription (Fig. 3c), or by using the pAHL15:GUS reporter (Fig. 3d,e), showed that AHL15 was indeed strongly upregulated in soc1 ful inflorescence nodes and lateral inflorescences. Previous studies have revealed that the expression of SOC1 is positively regulated by LD conditions. Moreover, SOC1 was shown to bind to the AHL15 upstream and downstream regions (Chr3, 20603158-20604316 and 20610947-20612012), which both contain a canonical CArGbox (CC[A/T]6GG) (Fig. 3f $)^{17-19}$. This, together with our own data, suggested that AHL15 expression is repressed by SOC1 under LD conditions (Fig. 2c) and that unrepressed AHL15 activity in the soc1 ful background explains the aerial rosette formation and 


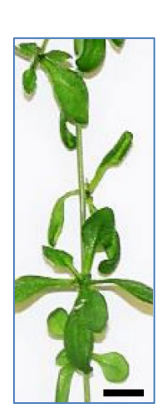

Col-0
Short day

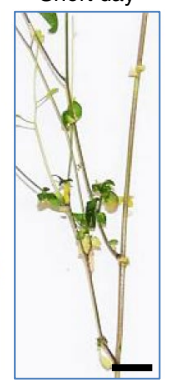

ah/15

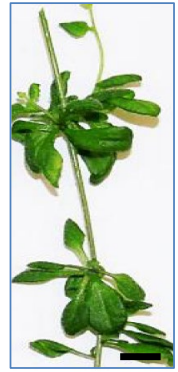

ahl15 pAHL15:AHL15

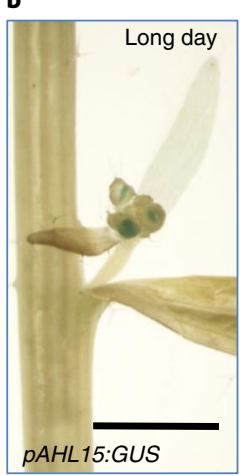

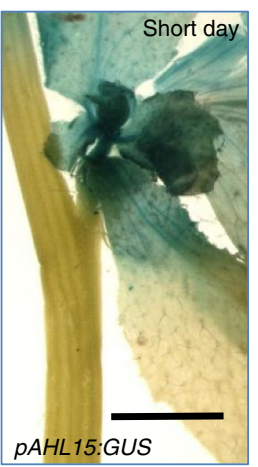

d

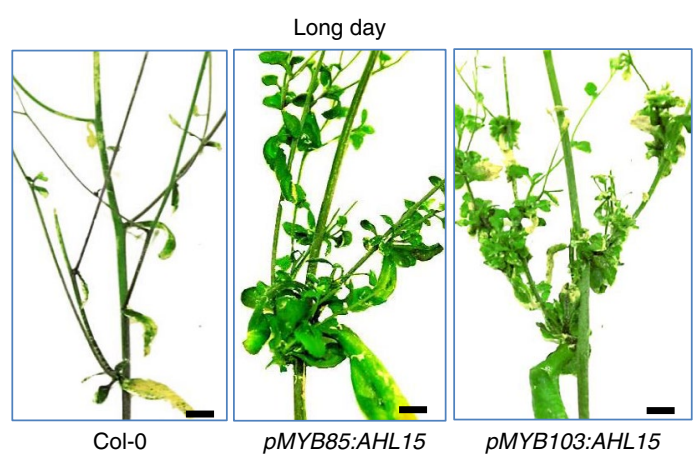

f

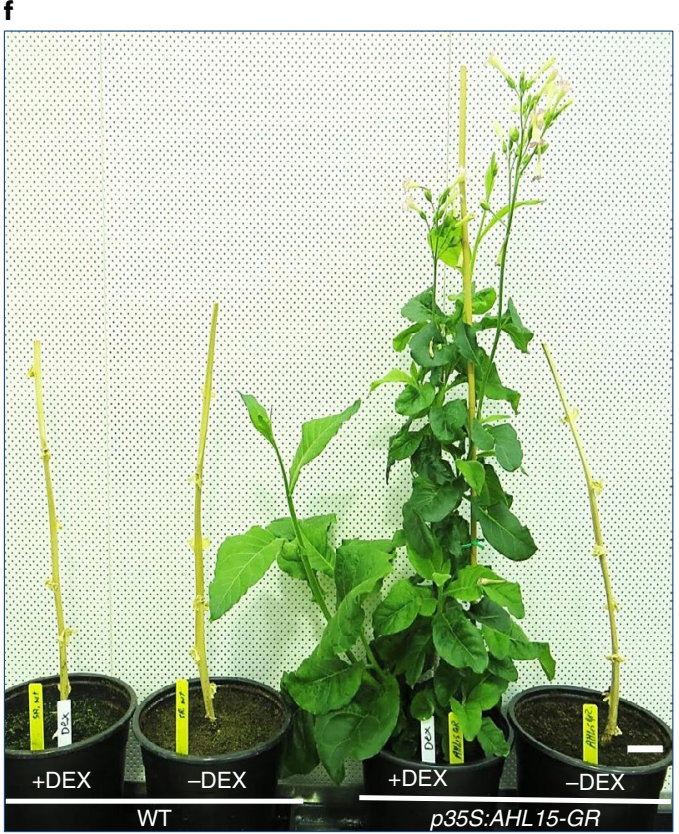

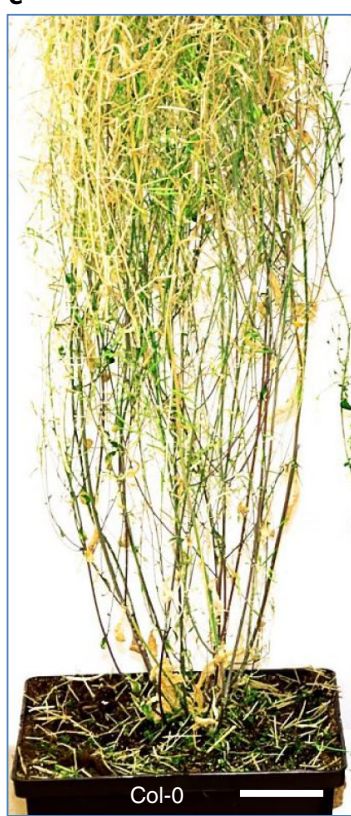

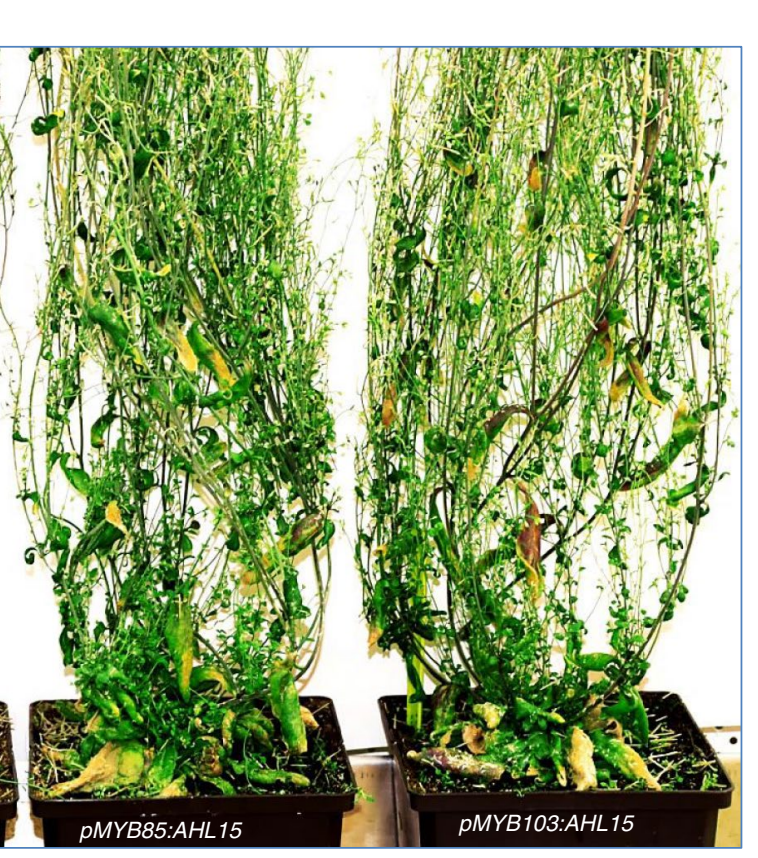
.

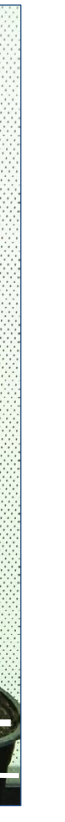

Fig. 2 | AHL15 promotes longevity in Arabidopsis and tobacco. a, Rosette leaves produced by aerial AMs in 4-month-old wild-type (left) and ah/15 pAHL15:AHL15 (right) plants, but not in ah/15 mutant plants (middle), grown under SD conditions. b,c, pAHL15:GUS expression in a lateral inflorescence of a 9-week-old plant grown under LD conditions (b) and a 4-month-old plant grown under SD conditions (c). d, Lateral aerial nodes without and with rosette leaves in 4-month-old wild-type (left), pMYB85:AHL15 (middle) and pMYB103:AHL15 (right) plants grown under LD conditions. e, Phenotype of

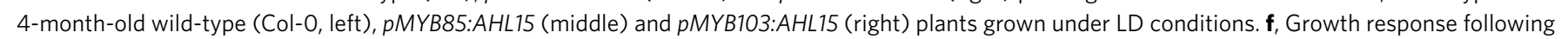
DEX treatment in bare stems of 5-month-old wild-type (WT, left) and 35S:AHL15-GR (right) tobacco plants grown under LD conditions. Scale bars, $1 \mathrm{~cm}$ in $\mathbf{a}-\mathbf{d}, 2 \mathrm{~cm}$ in $\mathbf{b}$ and $5 \mathrm{~cm}$ in $\mathbf{c}$. a-c,f, Similar results were obtained from two independent experiments. d,e, Similar results were obtained from three independent experiments.

polycarpic-like growth of mutant plants. To check whether the CArG-box-containing regions could also be bound by FUL, we used stem fragments containing axillary nodes of pFUL:FULGFP ful plants to perform chromatin immunoprecipitation (ChIP). Subsequent qPCR revealed significant enrichment for both upstream and downstream regions (Fig. 3g), indicating that FUL can repress AHL15 expression by direct binding to these regions. To further confirm that FUL and SOC1 can bind CArGboxes in the AHL15 up- and downstream regions, we performed electrophoretic mobility shift assay (EMSA) experiments. Probe fragments containing the corresponding regulatory regions (frag 1 and frag 3), or these regions with a mutated CArG-box (frags $1 \mathrm{~m}$ and $3 \mathrm{~m}$ ), were tested with SOC1 and FUL homo- and heterodimers. The SOC1/FUL heterodimer could bind to both regulatory fragments, but binding was reduced when the CArG-box was mutated in frag $1 \mathrm{~m}$ and even completely abolished in frag $3 \mathrm{~m}$ (Fig. 3h), providing additional evidence for the importance of the CArG-boxes in regard to the binding of SOC1 and FUL. The SOC1 homodimer showed the same results, while the FUL homodimer did not show binding (Supplementary Fig. 3).

SOC1 and FUL are known as central floral integrators, because they integrate the different environmental and endogenous signalling pathways that influence flowering ${ }^{13,17,20}$. They promote flowering through activation of the floral meristem genes APETALA1 (AP1) and LEAFY $(L F Y)^{21,22}$, and of genes involved in the biosynthesis of the plant hormone gibberellic acid (GA) ${ }^{23}$, which plays an important role in the promotion of flowering through activation of SOC1 and the SQUAMOSA PROMOTER BINDING PROTEIN-LIKE (SPL) genes ${ }^{24,25}$. Interestingly, AHL15 and AHL25 also control GA biosynthesis by direct binding to the promoter of GA3-oxidase1 (GA3OX1), which encodes an enzyme required for GA biosynthesis ${ }^{26}$. We therefore investigated the relationship between GA biosynthesis and AHL15 in the control of AM maturation. qPCR analysis showed that the expression of genes GA3OX1, GA20OX1 and GA20OX2, encoding rate-limiting enzymes in the final steps of the GA biosynthetic pathway ${ }^{23,27,28}$, 


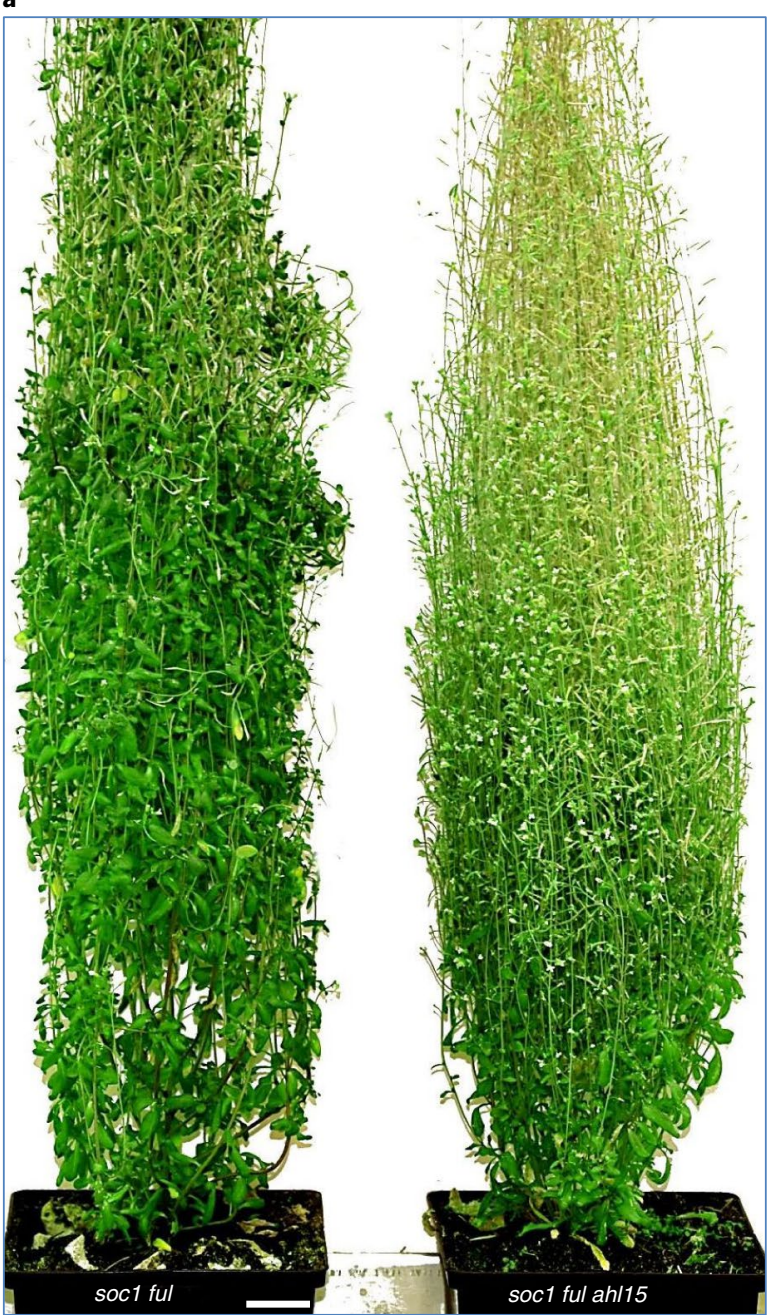

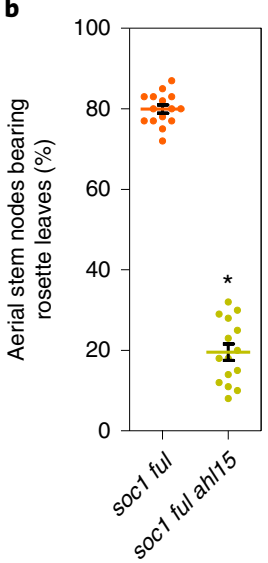

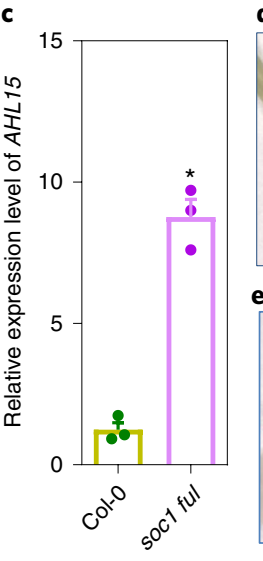

d

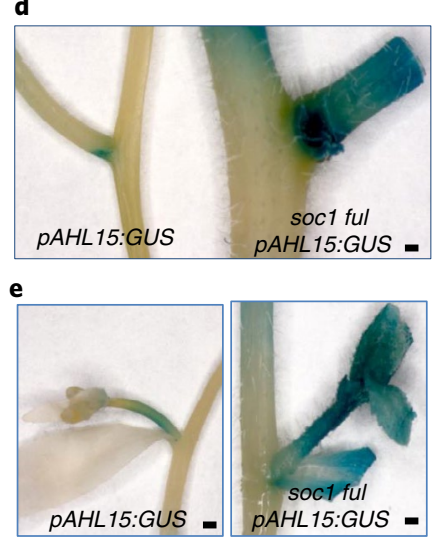

f

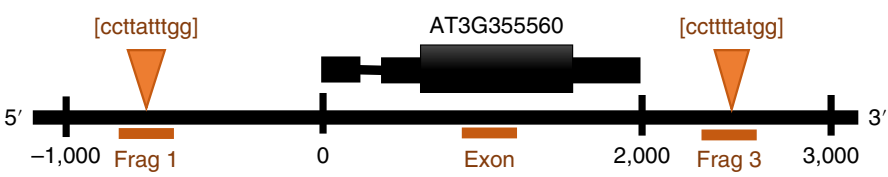

g

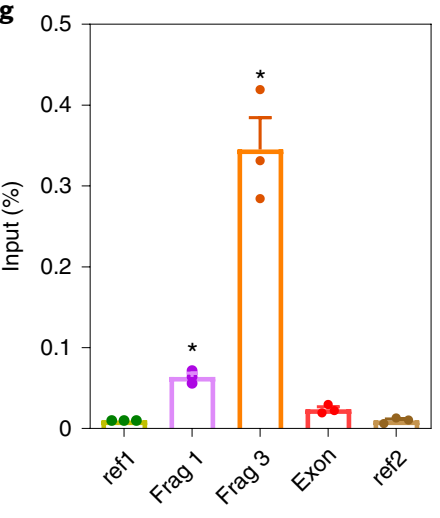

h

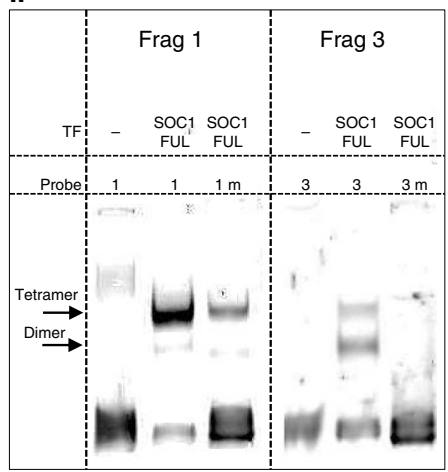

Fig. 3 | AHL15 is essential for suppression of AM maturation in the Arabidopsis soc1 ful mutant. a, A 3-month-old soc1 ful double-mutant plant with numerous aerial rosettes (left) and a soc1 ful ah/15 triple-mutant plant with a limited number of aerial rosettes (right), both grown under LD conditions. Similar results were obtained from two independent experiments. b. Percentage of aerial stem nodes bearing rosette leaves in 3-month-old soc 1 ful and soc1 ful ah/15 plants. Dots indicate the percentage per plant ( $n=10$ biologically independent plants), horizontal lines the mean and error bars indicate s.e.m. The asterisk indicates a significant difference $(P<0.001)$ as determined by two-sided Student's $t$-test. c, qPCR analysis of AHL15 expression in secondary inflorescence nodes of wild-type (Col-0) and soc 1 ful plants 2 weeks after flowering. Dots indicate the values of three biological replicates per plant line, bars indicate the mean and error bars indicate s.e.m. The asterisk indicates a significant difference $(P<0.001)$ as determined by two-sided Student's $t$-test. d,e, pAHL15:GUS expression in an inflorescence node (d) and a secondary inflorescence (e) in wild-type (left) or a soc1 ful mutant (right) background. Plants were at a comparable developmental stage, and similar results were obtained from two independent experiments. f, AHL15 gene model with canonical CArG-boxes located in the upstream (frag 1) and downstream (frag 3) regions, but not in the exon fragment that was used as control (exon). The AHL15 coding region is indicated by a thick black bar, exons by intermediate black bars and the position of the intron by a black line. $\mathbf{g}$, Graph showing ChIP-qPCR results from FUL-GFP ap1 cal secondary inflorescence nodes using an anti-green fluorescent protein antibody. Enrichment of fragments was calculated as a percentage of the input sample. ref1 and ref2 are reference fragments (see Methods), while other fragments are also indicated in the gene model. Dots indicate the values of three biological replicates, bars the means and error bars indicate s.e.m. Asterisks indicate significant differences with ref1 and ref2 $(P<0.001)$ as determined by two-sided Student's $t$-test. Exact $P$ values are provided in Supplementary Table 5 . $\mathbf{h}$, Binding of the transcription factors (TFs) SOC1 and FUL to regulatory regions near AHL15. Left: EMSA of promoter frag 1 with a wild-type (1) or mutated (m1) CArG-box. Right: EMSA of downstream frag 3 with a wild-type ( 3 ) or mutated $(3 \mathrm{~m}$ ) CArG-box. Shifting of the probe, indicating binding, occurred through either a tetramer (top band) or dimer (bottom band). Similar results were obtained from two independent experiments. Scale bars, $2 \mathrm{~cm}$ in $\mathbf{a}$ and $1 \mathrm{~mm}$ in $\mathbf{d , e}$.

was downregulated in DEX-treated 35S:AHL15-GR inflorescence nodes (Fig. 4a). In line with the downregulation of GA biosynthesis, GA application to DEX-treated flowering $p 35 S: A H L 15-G R$ plants resulted in marked repression of vegetative AM activity (Fig. 4b). In turn, treatment of flowering wild-type Arabidopsis plants by paclobutrazol, a potent inhibitor of GA biosynthesis, prevented AM maturation, resulting in aerial rosette leaf formation and enhanced longevity (Fig. 4c). Based on our findings, we postulate that AHL15 acts downstream of SOC1 and FUL as a central repressor of AM maturation, and that AHL15 prevents AM maturation in part by suppressing GA biosynthesis (Fig. 4d). Interestingly, the polycarpic behaviour of A. alpina was shown to be based on age-dependent suppression of AaSOC1 expression ${ }^{15}$ and GA levels ${ }^{29}$ and, as in our model (Fig. 4d), AHL genes may also link these two regulatory pathways thus facilitating polycarpic growth in A. alpina. 

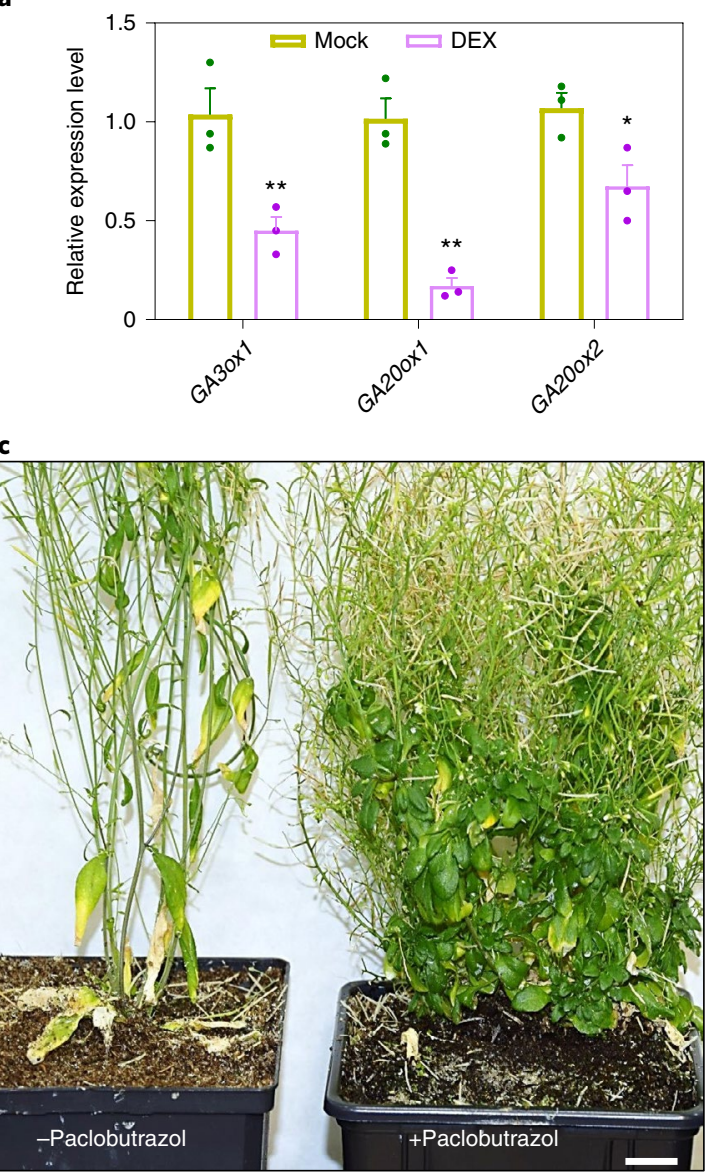

b

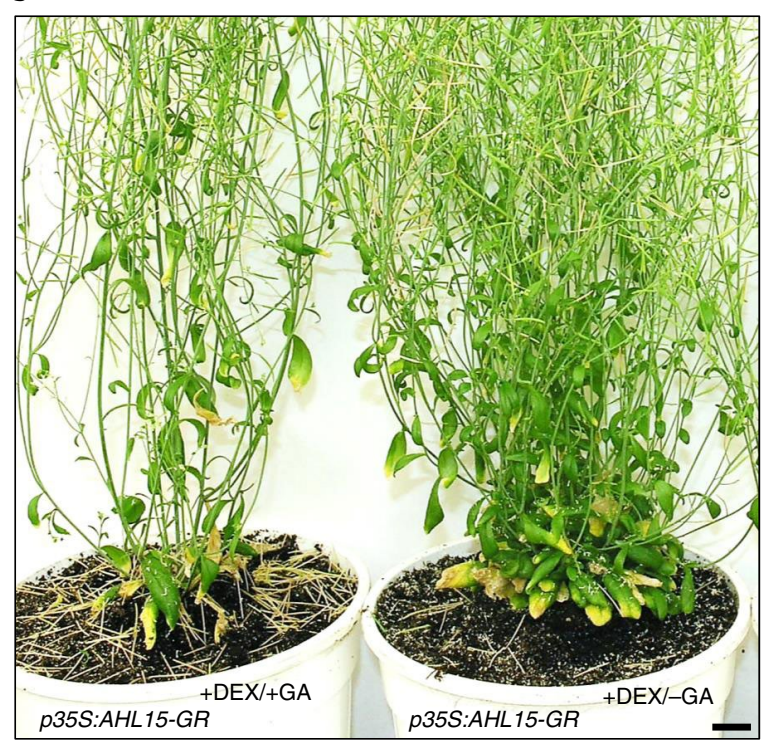

d

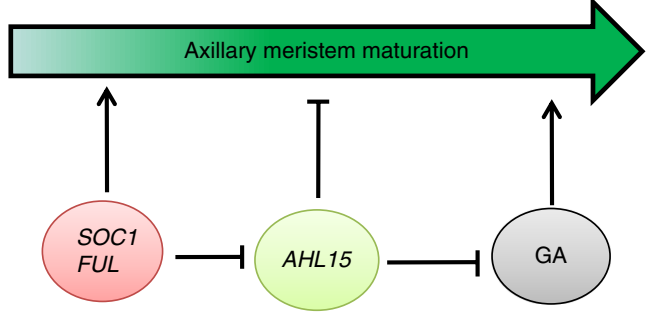

Fig. 4 | AHL15 delays AM maturation in part by suppression of GA biosynthesis. a, Relative expression level of GA biosynthesis genes GA3OX1, GA200X1 and GA200X2 by qPCR analysis in the basal regions of 1-week-old 35S:AHL15-GR inflorescences 1 day after spraying with either water (mock) or $20 \mu \mathrm{M}$ DEX. Dots indicate the values of three biological replicates per plant line, bars indicate the mean and error bars indicate s.e.m. Asterisks indicate significant differences from mock-treated plants $\left({ }^{\star} P<0.05\right.$, $\left.{ }^{\star \star} P<0.01\right)$, as determined by two-sided Student's $t$-test. $\mathbf{b}$, Shoot phenotype of 3-month-old $p 35 S$ : AHL15-GR plants that were DEX sprayed at 5 weeks of age and subsequently sprayed 1 week later with either $10 \mu \mathrm{M}$ GA ${ }_{4}(+\mathrm{GA})$ or water (-GA). Similar results were obtained from two independent experiments. c, Shoot phenotype of 3-month-old wild-type Arabidopsis plants that were sprayed 6 weeks earlier with either water (-Paclobutrazol) or $3 \mu \mathrm{M}$ paclobutrazol (+Paclobutrazol). Similar results were obtained from two independent experiments. Plants in $\mathbf{b}, \mathbf{c}$ were grown under LD conditions; scale bars, $2 \mathrm{~cm}$. d, Proposed model for the key role of AHL15 (and other AHL clade-A genes) in controlling AM maturation downstream of flowering genes SOC1 and FUL and upstream of GA biosynthesis. Blunt-ending lines indicate repression, arrows indicate promotion.

The existence of both mono- and polycarpic species within many plant genera indicates that life history traits have changed frequently during evolution ${ }^{5}$. AHL clade-A gene families can be found in both mono- and polycarpic plant species (Supplementary Fig. $4 \mathrm{a}$ ) ${ }^{30}$, and expression of the $A H L$ clade-A gene family could therefore provide a mechanism by which a plant species attains a polycarpic growth habit. Comparison of gene family size in representative species of three plant families did, however, not show obvious gene deletion or duplication events linked, respectively, to the mono- or polycarpic growth habit (Supplementary Fig. 4b). This suggests that a switch from mono- to polycarpic habit or vice versa could possibly be mediated by a change in gene regulation.

To find support for this, we compared the $A H L$ gene family of Arabidopsis with that of its close polycarpic relative A. lyrata ${ }^{31}$. The Arabidopsis and A. lyrata genomes both encode 15 AHL clade-A proteins, among which orthologous pairs can clearly be identified based on amino acid sequence identity (Supplementary Fig. 5). We hypothesized that the polycarpic habit of A. lyrata might be associated with enhanced $A H L$ clade-A gene expression leading to the maintenance of basal AMs in the vegetative state during flowering (Extended Data Fig. 10a). Analysis of individual AHL clade-A genes in Arabidopsis showed that expression of the majority of these genes, including AHL15, AHL19 and AHL20, was decreased in rosette nodes of Arabidopsis flowering plants compared with 2-week-old seedlings (Extended Data Fig. 10b). In contrast, the expression of five members of the $A H L$ gene family (AHL15, AHL17, AHL19, AHL20 and AHL27) was significantly higher in rosette nodes of flowering $A$. lyrata plants compared with seedlings (Extended Data Fig. 10c). These data are in line with our hypothesis, and suggest that the different life history strategies in Arabidopsis and A. lyrata may be associated with the differential regulation of $A H L$ genes in AMs.

In conclusion, our data provide evidence for a novel role for AHL15 and other AHL clade-A genes in suppression of AM maturation and enhancement of plant longevity. Arabidopsis plants with enhanced AHL15 expression show polycarpic-like growth, but their vegetative AMs lack the dormancy that is characteristic of reproductive cycles in perennial plants (for example, A. lyrata and A. alpina). The importance of the SOC1/FUL-AHL-GA pathway in perennial life history therefore requires further confirmation. Although the 
exact mode of action of AHL proteins is largely unknown, they are characterized as DNA-binding proteins and, like AT-hook proteins in animals, they seem to act through chromatin remodelling ${ }^{11,32}$. It has been shown that AHL22 represses FLOWERING LOCUS T (FT) expression by binding to the FT promoter, where it possibly modulates the epigenetic signature around its binding region ${ }^{12}$. Detailed studies on the chromatin configuration by approaches, such as chromosome conformation capture technologies ${ }^{33}$, should provide more insight into the mode of action of these plant-specific AT-hook motif proteins. One of the objectives of our future research will be to unravel the molecular mechanisms by which these proteins influence plant development.

\section{Methods}

Plant materials, growth conditions and phenotyping. All Arabidopsis mutant and transgenic lines used in this study were in a Columbia (Col-0) background. The ahl15 (SALK_040729) transfer DNA (tDNA) insertion mutant and the previously described soc1-6 ful-7 double mutant ${ }^{34}$ were obtained from the Nottingham Arabidopsis Stock Centre. Seeds were planted directly into soil in pots and germinated at $21^{\circ} \mathrm{C}, 65 \%$ relative humidity and a 16 - (LD) or 8 -h (SD) photoperiod. When seedlings were $10 \mathrm{~d}$ old, they were thinned to one seedling per pot by cutting the hypocotyls. To score for phenotypes including longevity, Col-0 wild-type, mutant or transgenic plants were transferred to larger pots about 3 weeks after flowering. $N$. tabacum cv SR1 Petit Havana (tobacco) plants were grown in medium-sized pots at $25^{\circ} \mathrm{C}, 70 \%$ relative humidity and a $16-\mathrm{h}$ photoperiod. In regard to DEX (Sigma-Aldrich) treatment, Arabidopsis and tobacco plants were sprayed with 20 or $30 \mu \mathrm{M}$ DEX, respectively. To test the effect of GA on AHL15GR activation by DEX treatment, 35-d-old flowering $p 35 S$ :AHL15-GR plants were first sprayed with $20 \mu \mathrm{M}$ DEX followed 1 week later by spraying with $10 \mu \mathrm{M}$ GA4 (Sigma-Aldrich). The production of rosette leaves, cauline leaves, flowers or fruits per rosette or aerial AM of 5-, 7-, 9- or 10-week-old plants was determined by dividing the total number of leaves or fruits produced by the number of active rosette or aerial AMs per plant. In regard to flowering time, the number of rosette leaves produced by the SAM was counted following bolting.

Plasmid construction and transgenic Arabidopsis lines. To generate the different promoter:AHL15 gene fusions, the complete AHL15 (AT3G55560) genomic fragment from ATG to stop codon was amplified from genomic DNA of Arabidopsis ecotype Columbia (Col-0) using PCR primers Gateway-AHL15-F and - $\mathrm{R}$ (Supplementary Table 1). The resulting fragment was inserted into pDONR207 via a $B P$ reaction. $L R$ reactions were carried out to fuse the $A H L 15$ coding region downstream of the $35 S$ promoter in destination vector pMDC32 (ref. ${ }^{35}$ ). Subsequently, the $35 S$ promoter was excised with $K p n I$ and $S p h I$ and replaced by the Gateway cassette ( $c c d B$ flanked by attP sequences) amplified from pMDC164 (ref. ${ }^{35}$ ) by the KpnI and SphI flanked primers (Supplementary Table 1), resulting in plasmid pGW-AHL15. To generate the constructs $p F D: A H L 15$, pMYB85:AHL15, $p M Y B 103: A H L 15$ and $p A H L 15: A H L 15,3-\mathrm{kb}$ regions upstream of the ATG start codon of genes FD (AT4G35900), MYB85 (AT4G22680), MYB103 (AT1G63910) and AHL15 were amplified from ecotype Columbia (Col-0) gDNA using the forward (F) and reverse (R) PCR primers indicated in Supplementary Table 1 . The resulting fragments were first inserted into pDONR207 by BP reaction, and subsequently cloned upstream of the AHL15 genomic fragment in destination vector pGW-AHL15 by LR reaction. To generate reporter constructs pAHL15:GUS, pMYB85:GUS and PMYB103:GUS, the corresponding promoter fragments were cloned upstream of the GUS gene in destination vector pMDC164 by LR reaction. To generate the $p A H L 15: A H L 15-\Delta G$ construct, a synthetic KpnISpeI fragment containing the $A H L 15$ coding region lacking the sequence encoding amino acids Gly-Arg-Phe-Glu-Ile-Leu in the C-terminal region (BaseClear) was used to replace the corresponding coding region in the PAHL15:AHL15 construct. To construct 35S::AHL15-GR, a synthetic PstI-XhoI fragment containing the AHL15-GR fusion (Shine Gene Molecular Biotech; see Supplementary File 1) was used to replace the $B B M-G R$ fragment in binary vector pSRS031 (ref. ${ }^{36}$ ). To generate the remaining overexpression constructs, full-length complementary DNA clones of AHL19 (AT3G04570), AHL20 (AT4G14465), AHL27 (AT1G20900) and AHL29 (AT1G76500) from Arabidopsis Col-0, AC129090 from M. trunculata cv Jemalong (MtAHL15) and Bo-Hook1 (AM057906) from B. oleracea var alboglabra (BoAHL15) were used to amplify the open reading frames using the primers indicated in Supplementary Table 1. The resulting fragments were cloned into plasmid pJET1/blunt (GeneJE PCR Cloning Kit, no. K1221), and subsequently transferred as NotI fragments to binary vector pGPTV 35S-FLAG ${ }^{37}$. All binary vectors were introduced into Agrobacterium tumefaciens strain AGL1 by electroporation ${ }^{38}$, and Arabidopsis Col-0 and ahl15 plants were transformed using the floral dip method $^{39}$.

Tobacco transformation. Round leaf discs were prepared from the lamina of the third and fourth leaves of 1-month-old, soil-grown tobacco plants. The leaf discs were surface sterilized by three washes with sterile water followed by incubation in $10 \%$ chlorine solution for $20 \mathrm{~min}$, and by four or five subsequent washes with sterile water ${ }^{40}$. The surface-sterilized leaf discs were syringe infiltrated with an overnight acetosyringone (AS)-induced culture of A. tumefaciens strain AGL1 containing binary vector pSRS031 (grown to $\mathrm{OD}_{600}=0.6$ in the presence of $100 \mu \mathrm{M}$ AS) carrying the $35 \mathrm{~S}::$ AHL15-GR construct, and co-cultivated for $3 \mathrm{~d}$ in the dark on co-cultivation medium (CCM) consisting of full-strength MS medium $^{41}$ with $3 \%$ (w/v) sucrose ( $\mathrm{pH} 5.8$ ) solidified with $0.8 \%(\mathrm{w} / \mathrm{v})$ Diachin agar and supplemented with $2 \mathrm{mgl}^{-1}$ of 6-benzylaminopurine, $0.2 \mathrm{mgl}^{-1}$ of 1-naphthaleneacetic acid and $40 \mathrm{mgl}^{-1}$ of AS. After co-cultivation, the explants were transferred to CCM supplemented with $15 \mathrm{mgl}^{-1}$ of phosphinothricin (ppt) for selection and $500 \mathrm{mgl}^{-1}$ of cefotaxime to kill Agrobacterium. Regeneration was carried out at $24^{\circ} \mathrm{C}$ in a $16-\mathrm{h}$ photoperiod. The regenerated transgenic shoots were rooted in large jars containing $100 \mathrm{ml}$ of hormone-free MS medium with $15 \mathrm{mgl}^{-1}$ of ppt and $500 \mathrm{mgl}^{-1}$ of cefotaxime. The rooted transgenic plants were transferred to soil and grown in a growth room at $25^{\circ} \mathrm{C}, 75 \%$ relative humidity and a 16-h photoperiod. All transgenic plants were checked for the presence of the tDNA insert by PCR, using genomic DNA extracted from leaf tissues by the cetyltrimethylammonium bromide method ${ }^{42}$

Histochemical staining and microscopy. Histochemical staining of transgenic lines for GUS activity was performed as described previously ${ }^{43}$. Tissues were stained for $4 \mathrm{~h}$ at $37^{\circ} \mathrm{C}$, followed by chlorophyll extraction and rehydration by incubation for $10 \mathrm{~min}$ in a graded ethanol series (75, 50 and 25\%). GUS-stained tissues were observed and photographed using a Leica MZ12 microscope equipped with a Leica DC500 camera.

qPCR analysis. RNA isolation was performed using a NucleoSpin RNA Plant kit (Macherey-Nagel). For qPCR analysis, $1 \mu \mathrm{g}$ of total RNA was used for cDNA synthesis with the iScript cDNA Synthesis Kit (BioRad). PCR was performed using the SYBR-Green PCR Master mix (SYBR Premix Ex Taq, Takara) and a CFX96 thermal cycler (BioRad). The Pfaffl method was used to determine relative expression levels ${ }^{44}$. Expression was normalized using $\beta$-TUBULIN-6 and EF1$A L P H A$ as reference genes. Three biological replicates were performed, with three technical replicates each. The primers used are described in Supplementary Table 2.

ChIP-qPCR experiment. For ChIP-qPCR analysis, three independent samples were harvested from secondary inflorescence nodes of $p F U L: F U L-G F P$ ful plants and processed as described in refs. ${ }^{45,46}$. Primer sequences used for ChIP-qPCR are detailed in Supplementary Table 2.

EMSA experiment. EMSA was performed as previously described ${ }^{47}$. The sequences of the probes used are detailed in Supplementary Table 2.

$A H L$ clade-A gene family data retrieval. Nucleotide and amino acid sequences for $A H L$ clade-A genes in A. thaliana (AtAHLs) were retrieved by Biomart from Ensembl Plants (plants.ensembl.org/index.html). For our study we selected 15 additional species from three major plant families-Brassicaceae, Solanaceae and Fabaceae. Initially more species were included, but some were excluded from the analysis (for example, A. alpina) for reasons described below. The amino acid sequences of A. thaliana, A. lyrata, B. oleracea, Brassica rapa, Solanum lycopersicum, Solanum tuberosum, M. truncatula and Glycine max were downloaded from Ensembl Plants (ftp://ftp.ensemblgenomes.org). The genomes of N. tabacum, Capsicum annuum and Brassica napus were downloaded from NCBI Genome (ftp://ftp.ncbi.nih.gov/genomes/), and the genomes of Phaseolus vulgaris, Capsella rubella, Capsella grandiflora, Boechera stricta and Eutrema salsugineum were downloaded from Phytozome (https://phytozome.jgi.doe.gov/pz/portal.html)

Building of profile hidden Markov models (HMMs) and hmmer searches. Whole-protein sequences of Arabidopsis AHL clade-A genes were used as a query, and BLASTP ${ }^{48}$ with an $e$-value set at 0.001 was used to search for $A H L$ clade-A genes in the remaining plant genomes. Only BLASTP hits with $>70 \%$ coverage and $70 \%$ sequence identity and with an intact single AT-hook motif and PPC domain were used for building of profile HMMs. We used MAFFT software ${ }^{49}$ with the FF-NS-i algorithm for construction of seed alignments, which were manually inspected to remove any doubtful sequences. To increase the specificity of the search, columns with many gaps or low conservation were excluded using the trimAl software ${ }^{50}$. We applied a strict non-gap percentage threshold of 80 or similarity score $<0.001$ such that at least $30 \%$ of the columns was conserved. At this point several species were excluded (for example, A. alpina) because of extensive gaps in the sequence alignment. Profile HMMs were built from multiple sequence alignment-aligned fasta files using hmmbuild, and subsequent searches against the remaining 16 genomes were carried out using hmmsearch from the HMMER 3.1b1 package $^{51}$. AHL proteins in plants consist of two closely resembling clades-clade $\mathrm{A}$ and clade B. AHL sequences were classified to the clade-A family based on a comparison with clade-B AHL sequences, where a hit with lower $e$-value for either clade A or clade B would correctly place the sequence in the corresponding clade (for example, a low $e$-value for clade A would place the sequence in clade A and vice versa). 
Phylogenetic reconstruction and reconciliation. Phylogenetic analysis was carried out using both maximum likelihood with PhyML ${ }^{52}$ and Bayesian inference implementing the Markov chain Monte Carlo algorithm with MrBayes ${ }^{53}$. For Bayesian inference, we specified the number of substitution types (nst) equal to 6 and the rate variation (rates) as invgamma. Invgamma states that a proportion of the sites is invariable while the rate for the remaining sites is drawn from a gamma distribution. These settings are equivalent to the GTR + I+ gamma model. Two independent analyses (nruns $=2$ ) of four chains (three heated and one cold) were run simultaneously for at least 10 million generations, sampling every 1,000 generations. Burn-in was set at $25 \%$. For clade-A AHLs the simulations were run for 10 million generations, sampling every 1,000 generations and convergence was reached at 0.016 . For maximum likelihood analysis, we used the default amino acid substitution model LG and the number of bootstrap replicates was specified as 100 .

Tree resolving, rearrangement and reconciliation were carried out using NOTUNG software ${ }^{54}$. NOTUNG uses duplication/loss parsimony to fit a gene (protein) tree to a species tree. The species tree was obtained using PhyloT (https:// phylot.biobyte.de/), which generates phylogenetic trees based on NCBI taxonomy. Tree editing/manipulations were performed using the R packages $\mathrm{APE}^{55}$ and GEIGER $^{56}$. We applied a strict threshold for rearrangement of $90 \%$, after which we performed reconciliation of the gene (protein) tree with the species tree.

Reconstruction of evolutionary scenario using Dollo parsimony method. Dollo parsimony principles are commonly exploited for two-state character traits. To classify branches as either gene losses or gene gains, we used the Dollo parsimony method which allows for unambiguous reconstruction of ancestral character states, because it is based on the assumption that a complex character that has been lost during evolution of a particular lineage cannot be regained.

Reporting Summary. Further information on research design is available in the Nature Research Reporting Summary linked to this article.

\section{Data availability}

All processed data are contained in the manuscript, the Extended Data or the Supplementary Information. Raw data and materials generated during this study are available upon reasonable request.

Received: 19 May 2019; Accepted: 11 March 2020;

Published online: 13 April 2020

\section{References}

1. Grbić, V. \& Bleecker, A. B. Axillary meristem development in Arabidopsis thaliana. Plant J. 21, 215-223 (2000).

2. Wang, B., Smith, S. M. \& Li, J. Genetic regulation of shoot architecture. Ann. Rev. Plant Biol. 69, 437-468 (2018).

3. Park, S. J., Eshed, Y. \& Lippman, Z. B. Meristem maturation and inflorescence architecture-lessons from the Solanaceae. Curr. Opin. Plant Biol. 17, 70-77 (2014).

4. Munné-Bosch, S. Do perennials really senesce? Trends Plant Sci. 13, 216-220 (2008).

5. Amasino, R. Floral induction and monocarpic versus polycarpic life histories. Genome Biol. 10, 228 (2009).

6. Melzer, S. et al. Flowering-time genes modulate meristem determinacy and growth form in Arabidopsis thaliana. Nat. Genet. 40, 1489-1492 (2008).

7. Kiefer, C. et al. Divergence of annual and perennial species in the Brassicaceae and the contribution of cis-acting variation at FLC orthologues. Mol. Ecol. 26, 3437-3457 (2017).

8. Zhao, J., Favero, D. S., Peng, H. \& Neff, M. M. Arabidopsis thaliana AHL family modulates hypocotyl growth redundantly by interacting with each other via the PPC/DUF296 domain. Proc. Natl Acad. Sci. USA 110 E4688-E4697 (2013)

9. Street, I. H., Shah, P. K., Smith, A. M., Avery, N. \& Neff, M. M. The AT-hook-containing proteins SOB3/AHL29 and ESC/AHL27 are negative modulators of hypocotyl growth in Arabidopsis. Plant J. 54, 1-14 (2008).

10. Xiao, C., Chen, F., Yu, X., Lin, C. \& Fu, Y.-F. Over-expression of an AT-hook gene, AHL22, delays flowering and inhibits the elongation of the hypocotyl in Arabidopsis thaliana. Plant Mol. Biol. 71, 39-50 (2009).

11. Ng, K.-H., Yu, H. \& Ito, T. AGAMOUS controls GIANT KILLER, a multifunctional chromatin modifier in reproductive organ patterning and differentiation. PLoS Biol. 7, e1000251 (2009).

12. Yun, J., Kim, Y.-S., Jung, J.-H., Seo, P. J. \& Park, C.-M. The AT-hook motif-containing protein AHL22 regulates flowering initiation by modifying FLOWERING LOCUS T chromatin in Arabidopsis. J. Biol. Chem. 287, 15307-15316 (2012)

13. Andrés, F. \& Coupland, G. The genetic basis of flowering responses to seasonal cues. Nat. Rev. Genet. 13, 627-639 (2012).

14. Ko, J., Han, K., Park, S. \& Yang, J. Plant body weight-induced secondary growth in Arabidopsis and its transcription phenotype revealed by wholetranscriptome profiling. Plant Physiol. 135, 1069-1083 (2004).
15. Bergonzi, S. et al. Mechanisms of age-dependent response to winter temperature in perennial flowering of Arabis alpina. Science 340, 1094-1097 (2013).

16. Zhou, C.-M. et al. Molecular basis of age-dependent vernalization in Cardamine flexuosa. Science 340, 1097-1100 (2013).

17. Lee, J. \& Lee, I. Regulation and function of SOC1, a flowering pathway integrator. J. Exp. Bot. 61, 2247-2254 (2010).

18. Immink, R. G. H. et al. Characterization of SOCl's central role in flowering by the identification of its upstream and downstream regulators. Plant Physiol. 160, 433-449 (2012).

19. Tao, Z. et al. Genome-wide identification of SOC1 and SVP targets during the floral transition in Arabidopsis. Plant J. 70, 549-561 (2012).

20. Matsoukas, I. G., Massiah, A. J. \& Thomas, B. Florigenic and antiflorigenic signaling in plants. Plant Cell Physiol. 53, 1827-1842 (2012).

21. Turnbull, C. Long-distance regulation of flowering time. J. Exp. Bot. 62, 4399-43413 (2011).

22. Song, Y. H., Ito, S. \& Imaizumi, T. Flowering time regulation: photoperiodand temperature-sensing in leaves. Trends Plant Sci. 18, 575-583 (2013).

23. Andrés, F. et al. SHORT VEGETATIVE PHASE reduces gibberellin biosynthesis at the Arabidopsis shoot apex to regulate the floral transition. Proc. Natl Acad. Sci. USA 111, E2760-E2679 (2014).

24. Yu, S. et al. Gibberellin regulates the Arabidopsis floral transition through miR156-targeted SQUAMOSA promoter binding-like transcription factors. Plant Cell 24, 3320-3332 (2012).

25. Wang, J. W. Regulation of flowering time by the miR156-mediated age pathway. J. Exp. Bot. 5, 4723-4730 (2014).

26. Matsushita, A., Furumoto, T., Ishida, S. \& Takahashi, Y. AGF1, an AT-hook protein, is necessary for the negative feedback of AtGA3ox1 encoding GA 3-oxidase. Plant Physiol. 143, 1152-1162 (2007).

27. Huang, S. et al. Overexpression of 20-Oxidase confers a gibberellinoverproduction phenotype in Arabidopsis. Plant Physiol. 118, 773-781 (1998).

28. Rieu, I. et al. The gibberellin biosynthetic genes AtGA20ox1 and AtGA20ox2 act, partially redundantly, to promote growth and development throughout the Arabidopsis life cycle. Plant J. 53, 488-504 (2008).

29. Tilmes, V. et al. Gibberellins act downstream of Arabis PERPETUAL FLOWERING1 to accelerate floral induction during vernalization. Plant Physiol. 180, 1549-1563 (2019).

30. Zhao, J., Favero, D. S., Qiu, J., Roalson, E. H. \& Neff, M. M. Insights into the evolution and diversification of the AT-hook Motif Nuclear Localized gene family in land plants. BMC Plant Biol. 14, 266 (2014).

31. Remington, D. L., Figueroa, J. \& Rane, M. Timing of shoot development transitions affects degree of perenniality in Arabidopsis lyrata (Brassicaceae). BMC Plant Biol. 15, 1-13 (2015).

32. Lim, P. O. et al. Overexpression of a chromatin architecture-controlling AT-hook protein extends leaf longevity and increases the post-harvest storage life of plants. Plant J. 52, 1140-1153 (2007).

33. Dekker, J., Marti-Renom, M. A. \& Mirny, L. A. Exploring the threedimensional organization of genomes: interpreting chromatin interaction data. Nat. Rev. Genet. 14, 390-403 (2013).

34. Wang, J.-W., Czech, B. \& Weigel, D. miR156-regulated SPL transcription factors define an endogenous flowering pathway in Arabidopsis thaliana. Cell 138, 738-749 (2009)

35. Karimi, M., Depicker, A. \& Hilson, P. Recombinational cloning with plant gateway vectors. Plant Physiol. 145, 1144-1154 (2007).

36. Passarinho, P. et al. BABY BOOM target genes provide diverse entry points into cell proliferation and cell growth pathways. Plant Mol. Biol. 68 225-237 (2008).

37. Becker, D., Kemper, E., Schell, J. \& Masterson, R. New plant binary vectors with selectable markers located proximal to the left T-DNA border. Plant Mol. Biol. 20, 1195-1197 (1992).

38. Den Dulk-Ras, A. \& Hooykaas J. P. Electroporation of Agrobacterium tumefaciens. Methods Mol. Biol. 55, 63-72 (1995).

39. Clough, S. J. \& Bent, aF. Floral dip: a simplified method for Agrobacteriummediated transformation of Arabidopsis thaliana. Plant J. 16, 735-743 (1998)

40. Baltes, N. J., Gil-Humanes, J., Cermak, T., Atkins, P. \& Voytas, D. F. DNA replicons for plant genome engineering. Plant Cell 26, 151163 (2014).

41. Murashige, T. \& Skoog, F. A revised medium for rapid growth and bio assays with tobacco tissue cultures. Physiol. Plant. 15, 473-497 (1962).

42. Doyle, J. J. Isolation of plant DNA from fresh tissue. Focus 12, 13-15 (1990).

43. Anandalakshmi, R. et al. A viral suppressor of gene silencing in plants. Proc. Natl Acad. Sci. USA 95, 13079-13084 (1998).

44. Pfaffl, M. W. A new mathematical model for relative quantification in real-time RT-PCR. Nucleic Acids Res. 29, e45 (2001).

45. Mourik, H. Van, Muiño, J. M., Pajoro, A., Angenent, G. C. \& Kaufmann, K. Characterization of in vivo DNA-binding eents of plant transcription factors by ChIP-seq: experimental potocol and computational analysis. Methods Mol. Biol 1284, 93-121 (2015).

46. Balanzà, V. et al. Genetic control of meristem arrest and life span in Arabidopsis by a FRUITFULL-APETALA2 pathway. Nat. Commun. 9, 565 (2018). 
47. Bemer, M. et al. FRUITFULL controls SAUR10 expression and regulates Arabidopsis growth and architecture. J. Exp. Bot. 68, 3391-3403 (2017).

48. Altschup, S. F., Gish, W., Pennsylvania, T. \& Park, U. Basic local alignment search tool. J. Mol. Biol. 215, 403-410 (1990).

49. Katoh, K., Misawa, K., Kuma, K. \& Miyata, T. MAFFT: a novel method for rapid multiple sequence alignment based on fast Fourier transform. Nucleic Acids Res. 30, 3059-3066 (2002).

50. Capella-gutiérrez, S., Silla-martínez, J. M. \& Gabaldón, T. TrimAl: a tool for automated alignment trimming in large-scale phylogenetic analyses. Bioinformatics 25, 1972-1973 (2009).

51. Eddy, S. R. Accelerated profile HMM searches. PLoS Comp. Biol. 7 e1002195 (2011).

52. Guindon, S. \& Gascuel, O. A simple, fast, and accurate algorithm to estimate large phylogenies by maximum likelihood. Syst. Biol. 5, 696-704 (2003).

53. Ronquist, F. \& Huelsenbeck, J. P. MrBayes 3: Bayesian phylogenetic inference under mixed models. Bioinformatics 19, 1572-1574 (2003).

54. Liebert, M. A., Chen, K., Durand, D., Farach-colton, M. \& Al, C. E. T. NOTUNG: a program for dating gene duplications. J. Comp. Biol. 7, 429-447 (2000).

55. Paradis, E., Claude, J. \& Strimmer, K. APE: analyses of phylogenetics and evolution in R language. Bioinformatics 20, 289-290 (2004).

56. Harmon, L. J. et al. GEIGER: investigating evolutionary radiations. Bioinformatics 24, 129-131 (2008).

\section{Acknowledgements}

We thank K. Boutilier and T. Greb for critical comments on the manuscript. O.K. was financially supported by a grant from the Iran Ministry of Science, Research and Technology (no. 89100156), and subsidies were provided by Generade and the Building Blocks of Life research programme (no. 737.016.013, to R.O.), which is (partly) financed by the Dutch Research Council (NWO). M.K. was financially supported by a fellowship of the Institute of Biotechnology \& Genetic Engineering at the Agricultural University of Peshawar with financial support by the Higher Education Commission of Pakistan. R.R.H. was financially supported by the KU Leuven Research Fund. M.B. was supported by grant no. ALWOP.199, which is (partly) financed by NWO. P.M. and M.C. were supported in part by Innovation Subsidy Collaborative Projects (no. IS054064) from the Dutch Ministry of Economic Affairs.

\section{Author contributions}

O.K. and R.O. conceived and supervised the project. All authors designed the experiments and analysed and interpreted the results. O.K. and A.R. performed the majority of the Arabidopsis experiments, with contributions from M.B., P.M. and M.C. M.K. generated and analysed the tobacco lines. R.R.H. and V.N. analysed the $A H L$ gene families in mono- and polycarpic plant species. O.K. and R.O. wrote the manuscript.

All authors read and commented on versions of the manuscript.

\section{Competing interests}

The authors declare no competing interests.

\section{Additional information}

Extended data is available for this paper at https://doi.org/10.1038/s41477-020-0637-z. Supplementary information is available for this paper at https://doi.org/10.1038/ s41477-020-0637-z.

Correspondence and requests for materials should be addressed to R.O.

Peer review information Nature Plants thanks Richard Amasino and the other, anonymous, reviewers for their contribution to the peer review of this work.

Reprints and permissions information is available at www.nature.com/reprints. Publisher's note Springer Nature remains neutral with regard to jurisdictional claims in published maps and institutional affiliations.

(c) The Author(s), under exclusive licence to Springer Nature Limited 2020 

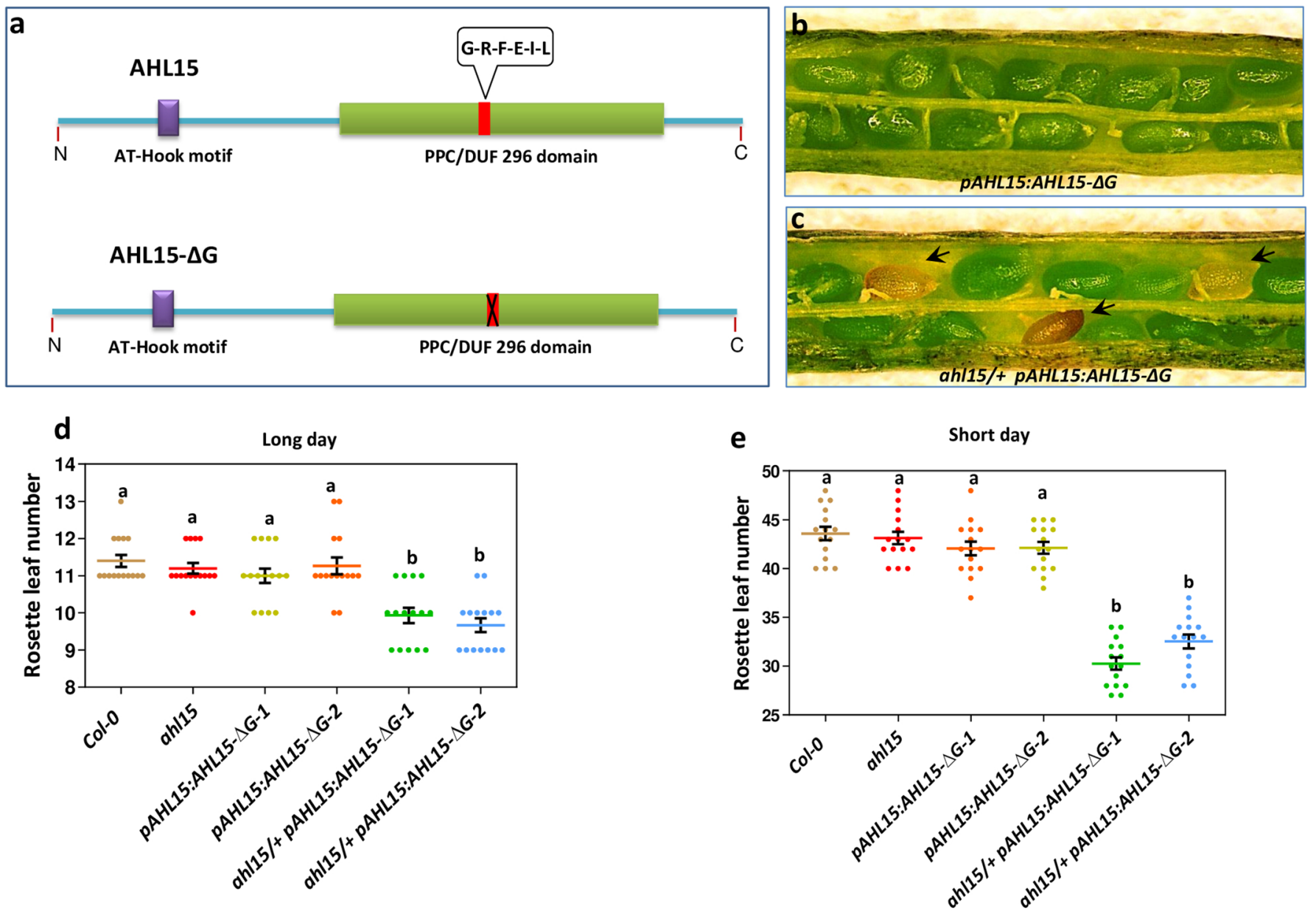

Extended Data Fig. 1 | Expression of a dominant negative AHL15- $\Delta$ G mutant protein. Expression of a dominant negative AHL15- $\Delta$ G mutant protein in the Arabidopsis ahl15 mutant background causes early flowering and impairs seed development. $\mathbf{a}$. The schematic domain structure of AHL15 and the dominant negative AHL15- $\Delta$ G version, in which six-conserved amino-acids (Gly-Arg-Phe-Glu-lle-Leu, red box) are deleted from the C-terminal PPC domain. b. Wild-type seed development in $p A H L 15: A H L 15-\Delta G$ siliques. c. Aberrant seed development (arrowheads) in ahl15/+pAHL15:AHL15- $\Delta G$ siliques (observed in 3 independent $P A H L 15: A H L 15-\Delta G$ lines crossed with the ah/15 mutant). Similar results were obtained in three independent experiments. d, e. The number of rosette leaves produced by the SAM in wild-type, ah/15, pAHL15:AHL15- $\Delta$ G and ah/15/+ pAHL15:AHL15- $\Delta$ G plants grown in long day (LD, d) or short day (SD, e) conditions. Two independent transgenic lines (1 and 2 ) were used in each experiment. Dots in $\mathbf{d}$ and $\mathbf{e}$ indicate the rosette leaf number per plant ( $n=15$ biologically independent plants), horizontal lines the mean and error bars the s.e.m. Letters ( $a, b, c)$ indicate statistically significant differences $(P<0.01)$, as determined by a one-way ANOVA with a Tukey's HSD post hoc test. The $P$ values can be found in the Supplementary Tables 6 and 7. 
a
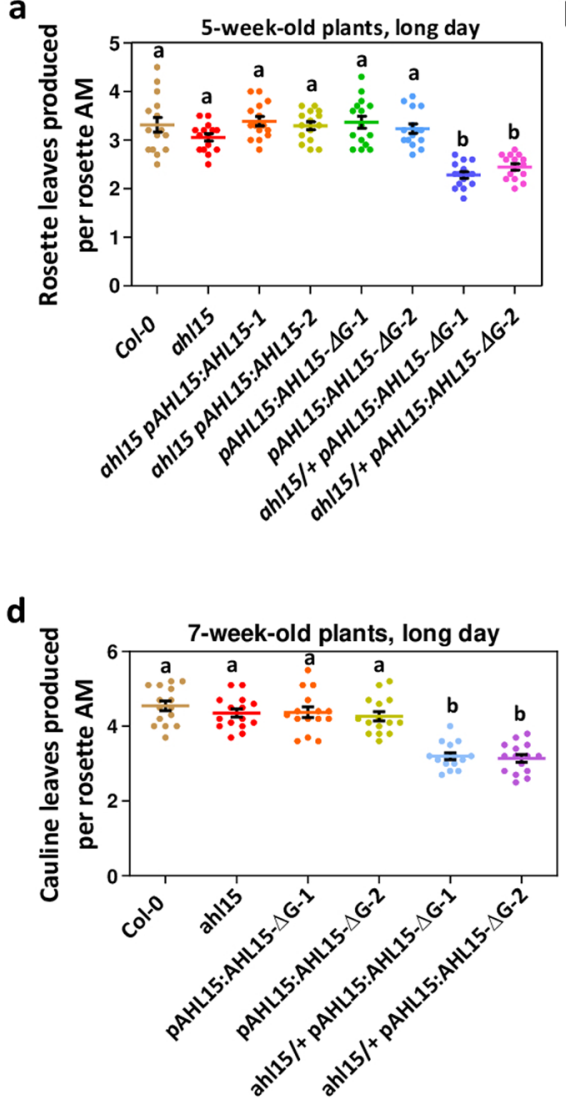

b
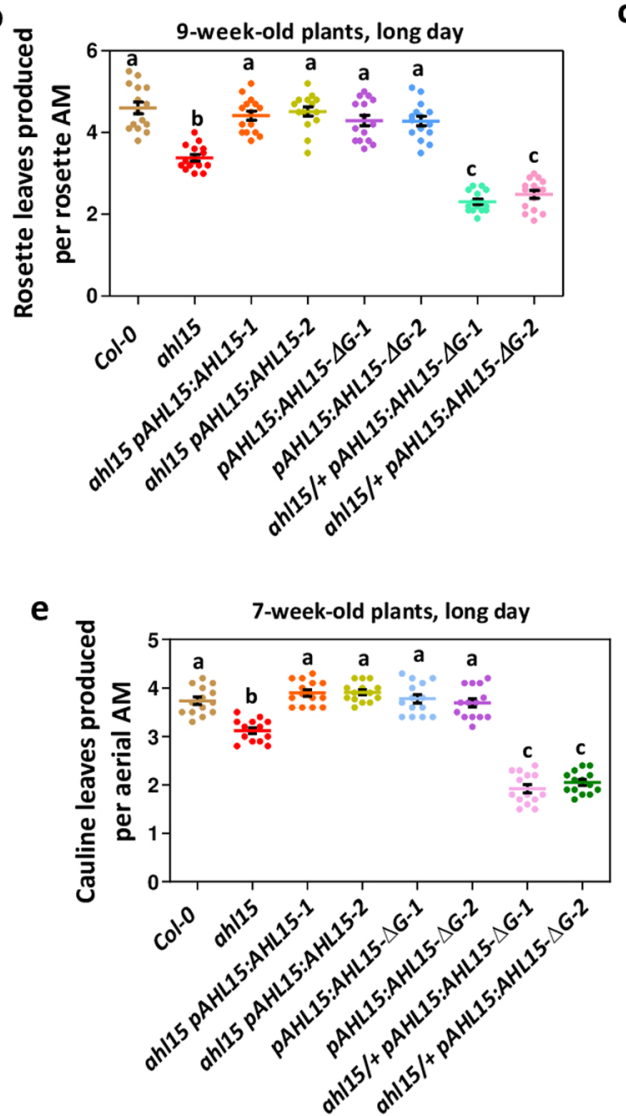

C
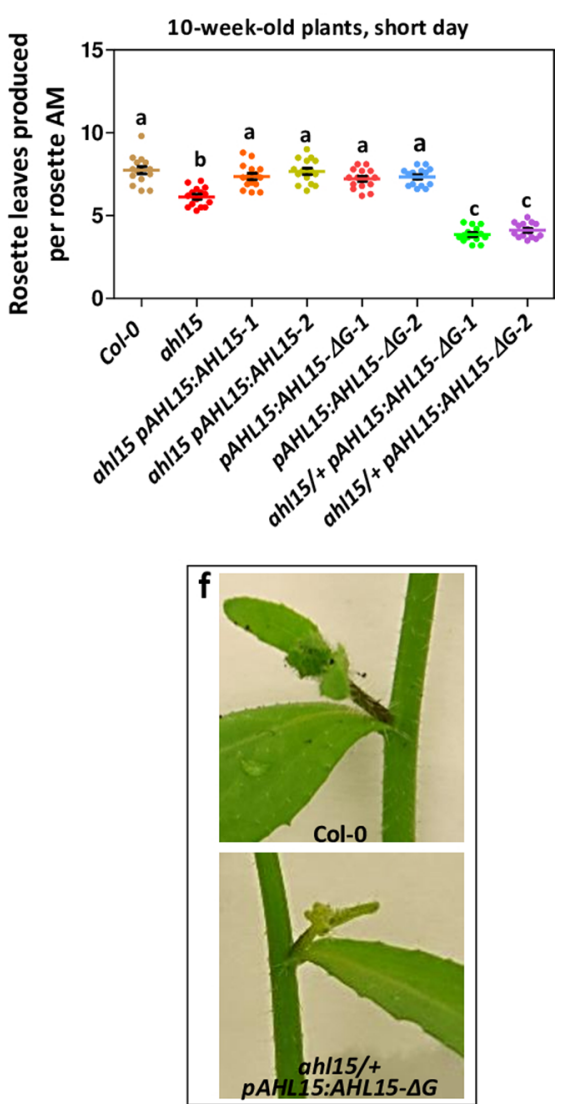

Extended Data Fig. 2 | AHL15 and other clade A AHL genes represses AM maturation in Arabidopsis. a, b. The number of rosette leaves produced per rosette AM of wild-type, ah/15, ahl15 pAHL15:AHL15, pAHL15:AHL15- $\Delta$ G and ahl15/+pAHL15:AHL15- $\Delta$ G plants 5 (a), 9 (b), or 10 weeks (c) after germination in long day $(L D, \mathbf{a}, \mathbf{b})$ or short day $(S D, \mathbf{c})$ conditions. d, e. The number of cauline leaves produced by rosette AMs (d) or by aerial AMs (e) of 7-week-old wild-type, ahl15, ahl15 pAHL15:AHL15, pAHL15:AHL15- $\Delta$ G and ah/15/+ pAHL15:AHL15- $\Delta$ G plants. Dots in a-e indicate rosette or cauline leaf number per AM per plant ( $n=15$ biologically independent plants), horizontal lines the mean, and error bars the s.e.m. Letters ( $a, b, c)$ indicate statistically significant differences $(P<0.01)$, as determined by a one-way ANOVA with a Tukey's HSD post hoc test. The $P$ values are provided in Supplementary Tables 8-12. f. A lateral inflorescence with cauline leaves formed on the first inflorescence node of a wild-type (top) or ahl15/+ pAHL15:AHL15- $\Delta$ G plant (bottom). Similar results were obtained in three independent experiments. 
a

Activity of rosette AMs on 6-week-old plants

ahl15

ahl15/+

PAHL15:AHL15- $\triangle G-1$
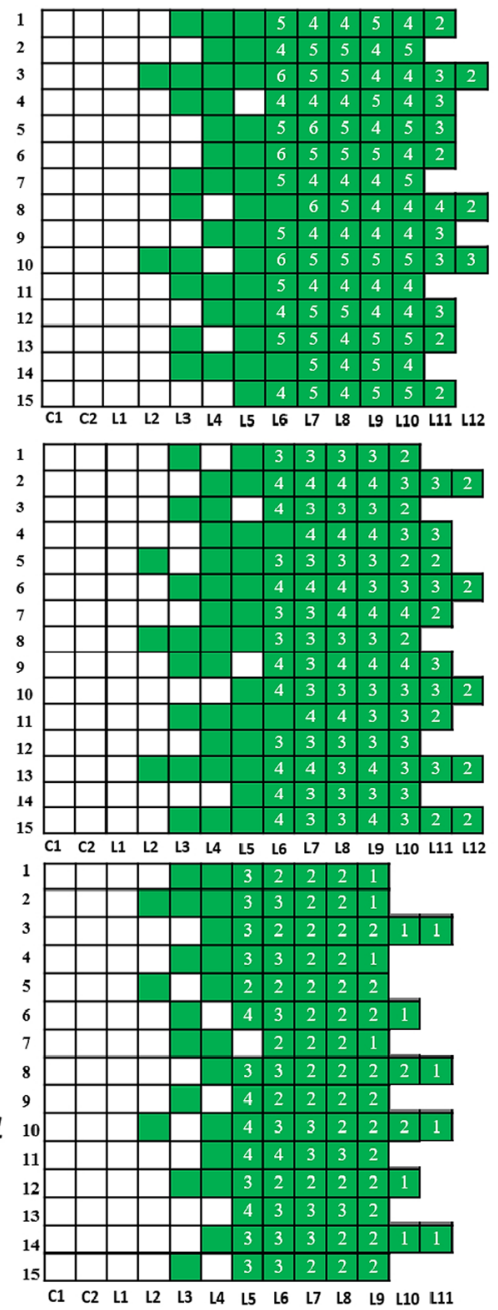

$\square$ no or inactive AM $\square$ Active AM

Numbers: rosette leaves produced per AM b Developmental phase of rosette AMs on 6-week-old plants

Col-0

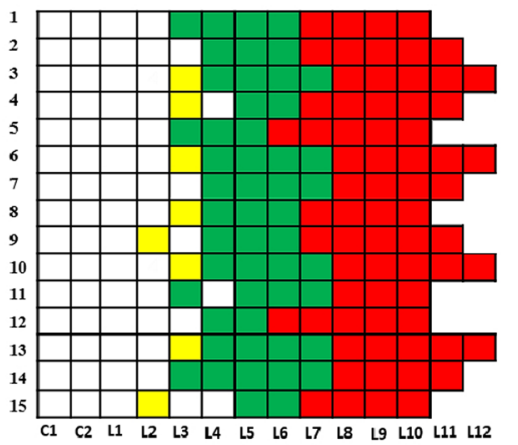

$a h / 15$

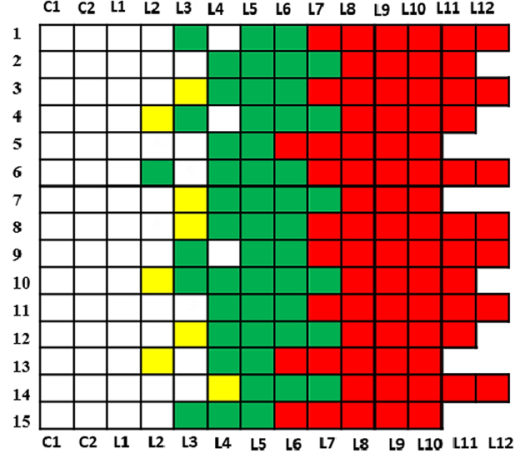

ahl15/t+

PAHL15:AHL15- $\triangle G-1$

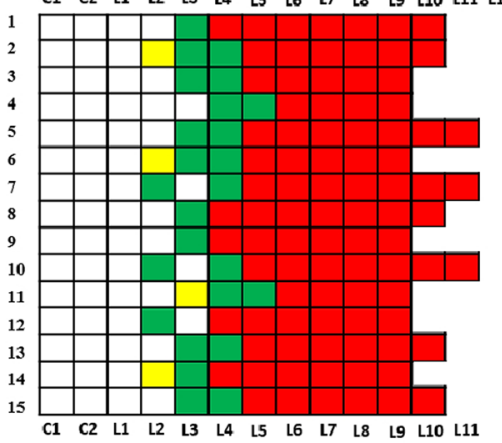

$\square$ no AM

AM with Leaf primordium

Vegetative phase $\square$ Reproductive phase

Extended Data Fig. 3 | Arabidopsis AHL genes enhance the vegetative activity and suppress the floral transition of rosette AMs. a. Schematic representation of the vegetative activity of rosette AMs of six-week-old wild-type, ah/15 and ah/15/+pAHL15:AHL15- $\Delta$ G-1 plants. Each row represents a single plant, and each square represents an individual AM in a cotyledon axil (C1 and C2) or in a rosette leaf axils (L1 to L12). The numbers within a square represent the number of rosette leaves produced by a rosette AM. A green square indicates a leaf axil with an active AM, as indicated by bud outgrowth or leaf development, and a white square indicates a leaf axil without an (active) AM. b. Developmental phase of the rosette AMs of six-week-old wild-type, ah/15 and ah/15/+ pAHL15:AHL15- $\Delta$ G-1 plants. White, yellow, green or red squares indicate axils without (active) AM, or rosette AMs with at least one visible leaf primordium, producing rosette leaves (vegetative) or producing cauline leaves or flowers (reproductive), respectively. Plants in $\mathbf{a}$ and $\mathbf{b}$ were grown in LD conditions. 

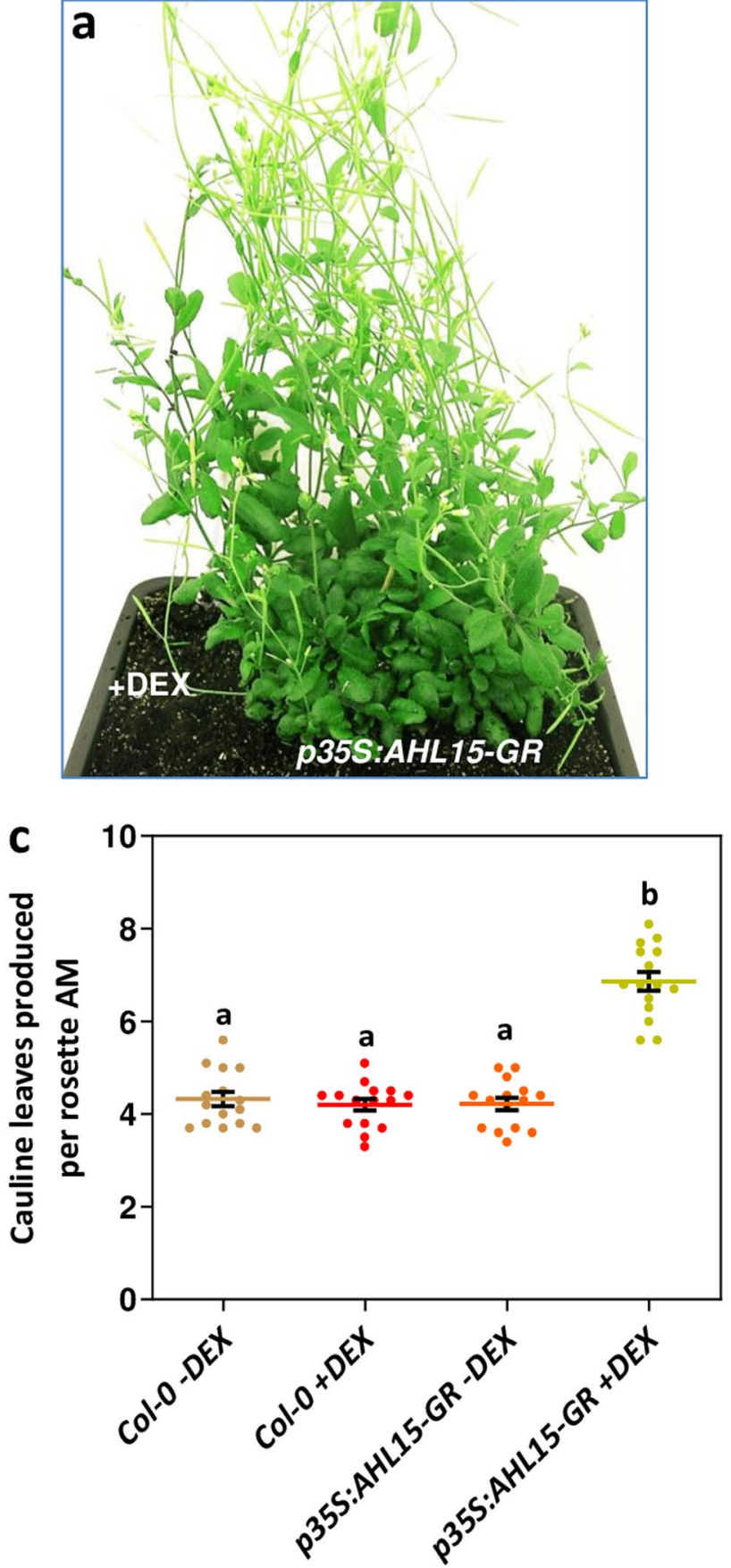
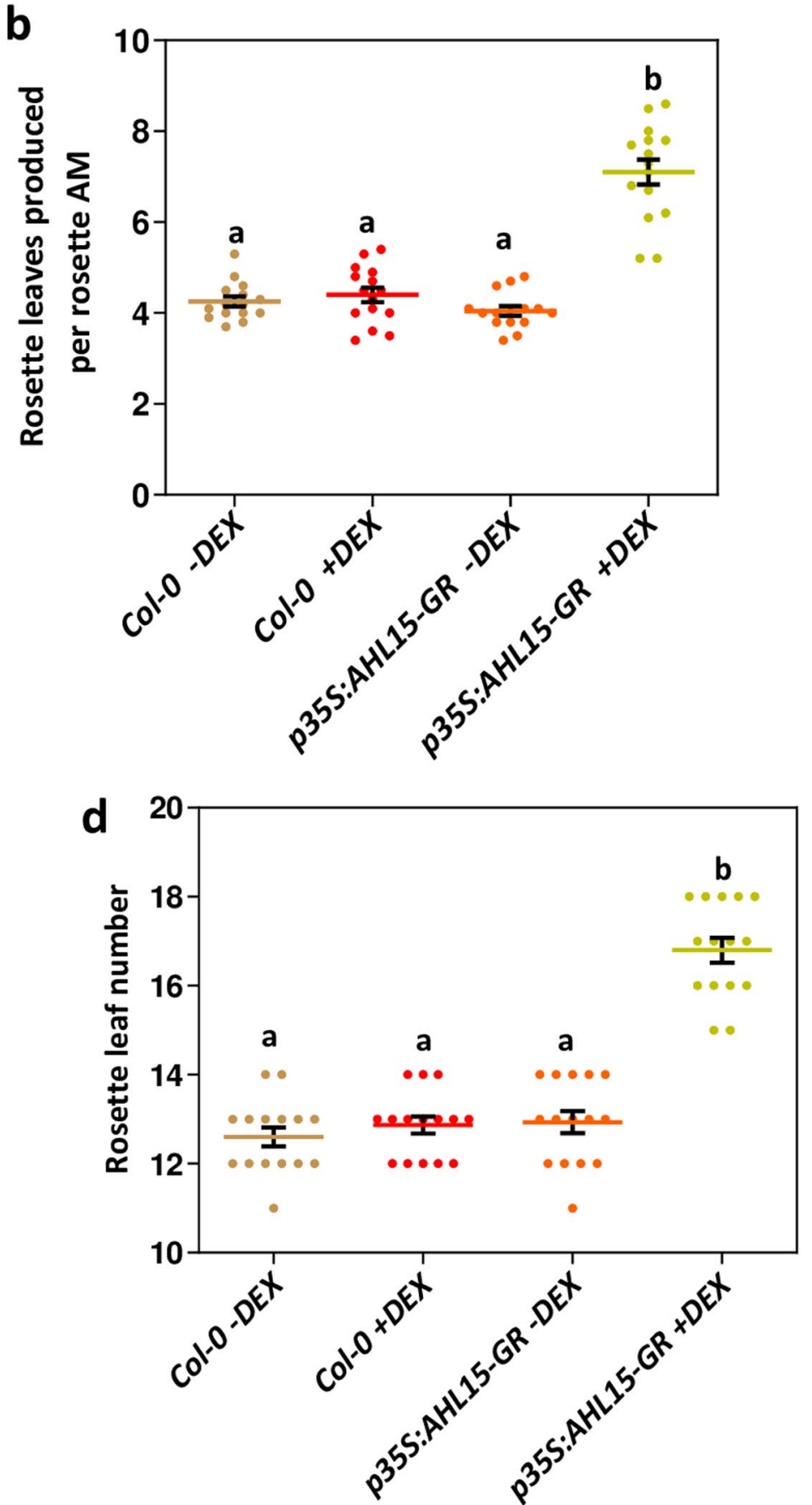

Extended Data Fig. 4 | AHL15 overexpression delays floral transition of the SAM and represses AM maturation. a. Shoot phenotype of a flowering 7-week-old 35S:AHL15-GR plant that was DEX-treated upon bolting (5 weeks old). Similar results were obtained in four independent experiments. b, c. Number of rosette leaves (b) or cauline leaves (c) produced by rosette AMs of 7-week-old mock-treated wild-type, DEX-treated wild-type, mocktreated 35S:AHL15-GR and DEX-treated 35S:AHL15-GR plants. Plants were DEX-treated upon bolting (5 weeks old) and scored 2 weeks later. d. The number of rosette leaves produced by the SAM in mock-treated wild-type, DEX-treated wild-type, mock-treated 35S:AHL15-GR and DEX-treated 35S:AHL15-GR plants. Non-flowering (3-week-old) plants were treated and the SAM-produced rosette leaves were counted after bolting. Dots in $\mathbf{b}$-d indicate number of leaves (per AM or SAM) per plant ( $n=15$ biologically independent plants), horizontal lines the mean, and error bars the s.e.m. Letters ( $a, b, c)$ indicate statistically significant differences $(P<0.01)$, as determined by a one-way ANOVA with a Tukey's HSD post hoc test. The $P$ values are provided in Supplementary Tables 13-15. Plants were grown in LD conditions. 

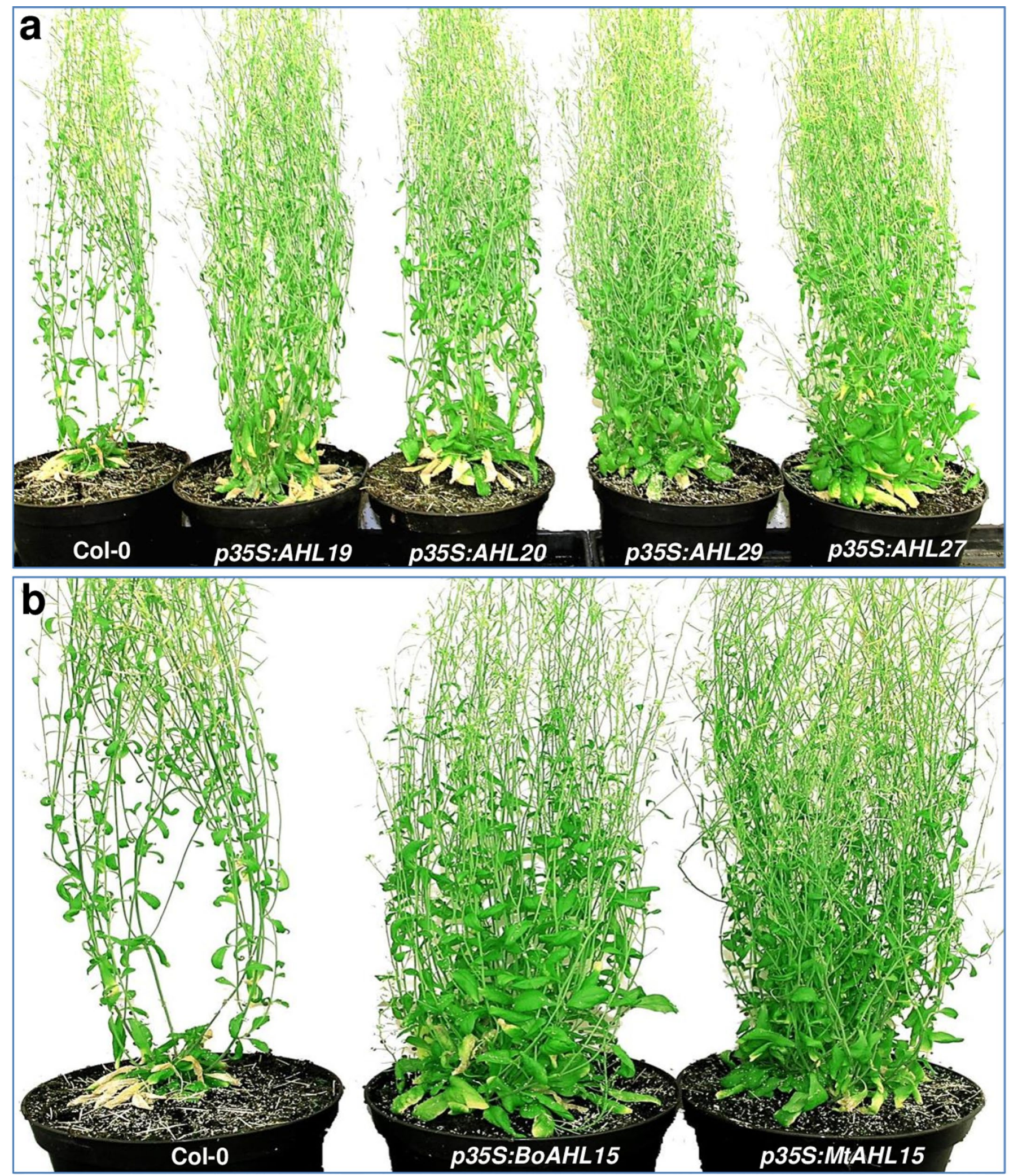

Extended Data Fig. 5 | Overexpression of Arabidopsis AHL15 paralogs or putative orthologs represses AM maturation in Arabidopsis. Overexpression of Arabidopsis AHL15 paralogs or putative orthologs represses AM maturation in Arabidopsis. (a and $\mathbf{b})$ Wild-type (Col-0) or transgenic 7-week-old Arabidopsis plants overexpressing Arabidopsis AHL19, AHL20, AHL27 and AHL29 (a), or the putative AHL15 orthologs from Brassica oleracea (BoAHL15) or Medicago trunculata (MtAHL15) (b). For a and $\mathbf{b}$ similar results were obtained in two independent experiments. Plants were grown in LD conditions. For presentation purposes, the original background of the images was replaced by a homogeneous white background. 


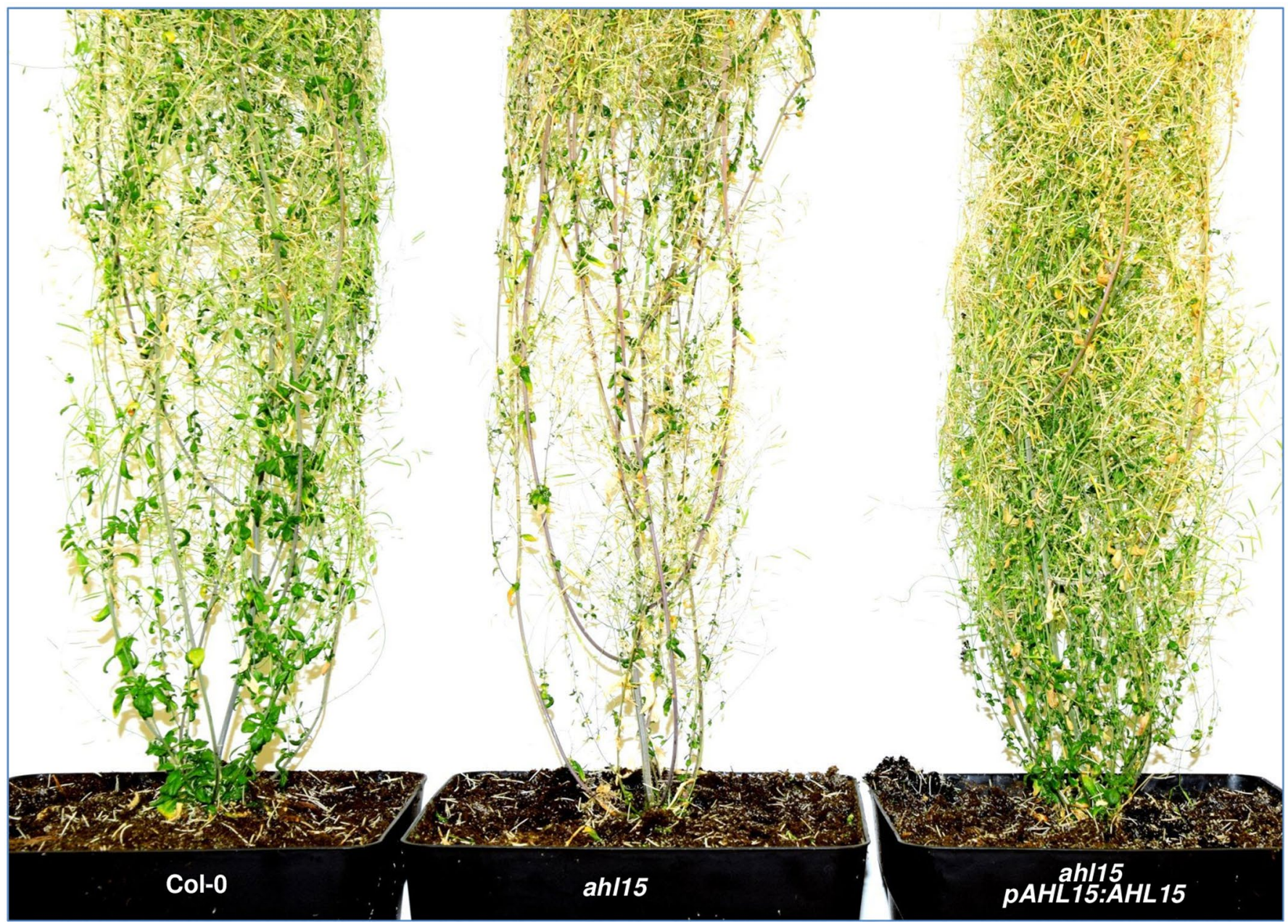

Extended Data Fig. 6 | AHL15 enhances the longevity of short day-grown Arabidopsis plants. Phenotype of 5-month-old wild-type (Col-0, left), ah/15 (middle) and ah/15 pAHL15:AHL15 (right) plants. The plants were grown in SD conditions. Similar results were obtained in two independent experiments. For presentation purposes, the original background of the image in was replaced by a homogeneous white background. 


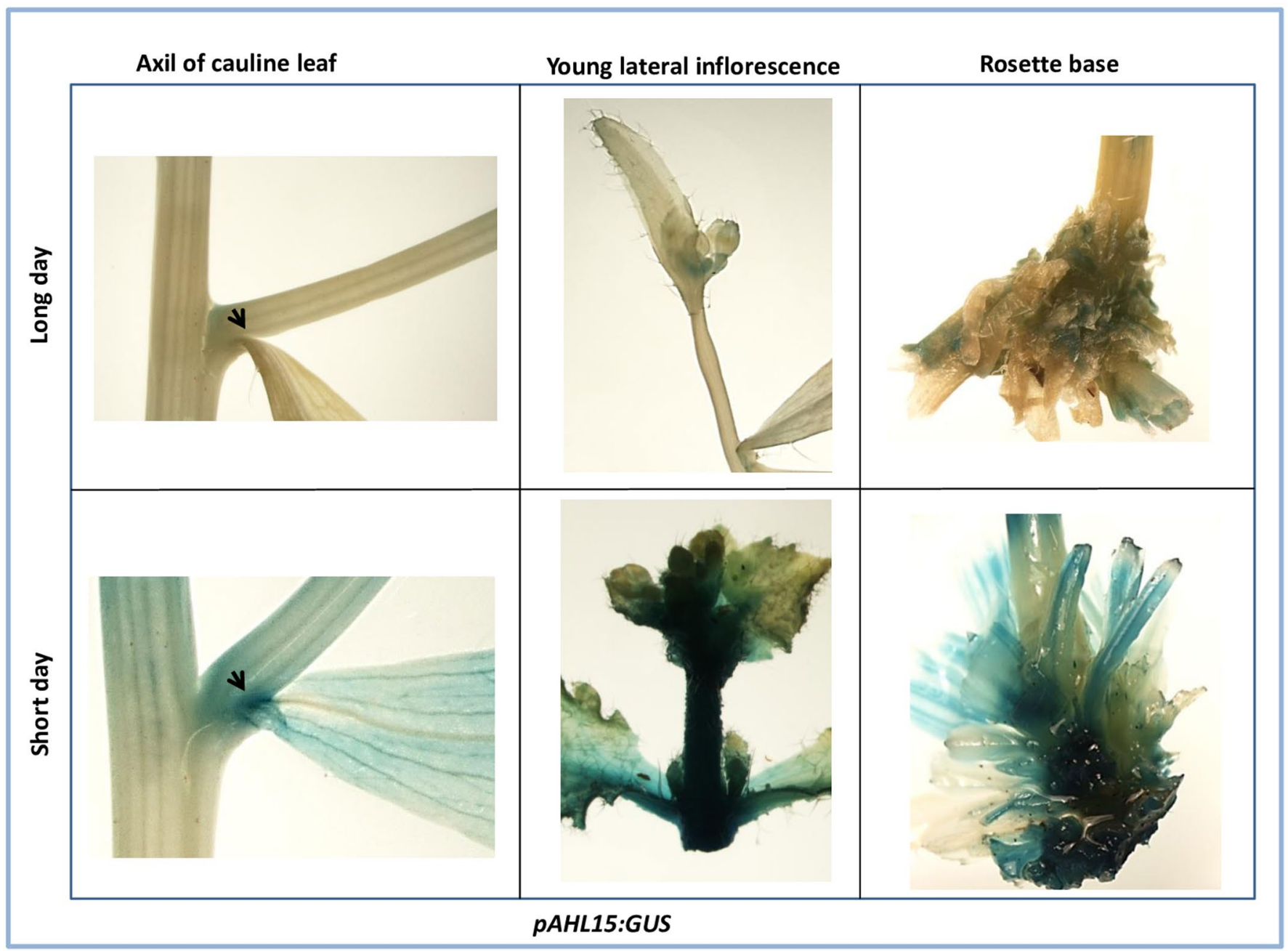

Extended Data Fig. 7 | AHL15 expression is day length sensitive. Expression of the pAHL15:GUS reporter in the axil of a cauline leaf (left, arrowheads), Young lateral inflorescence (middle) and rosette base (right) of a 9-week-old plant grown in LD conditions (top) or a 4-month-old plant grown under SD conditions (bottom). Similar results were obtained in two independent experiments. 


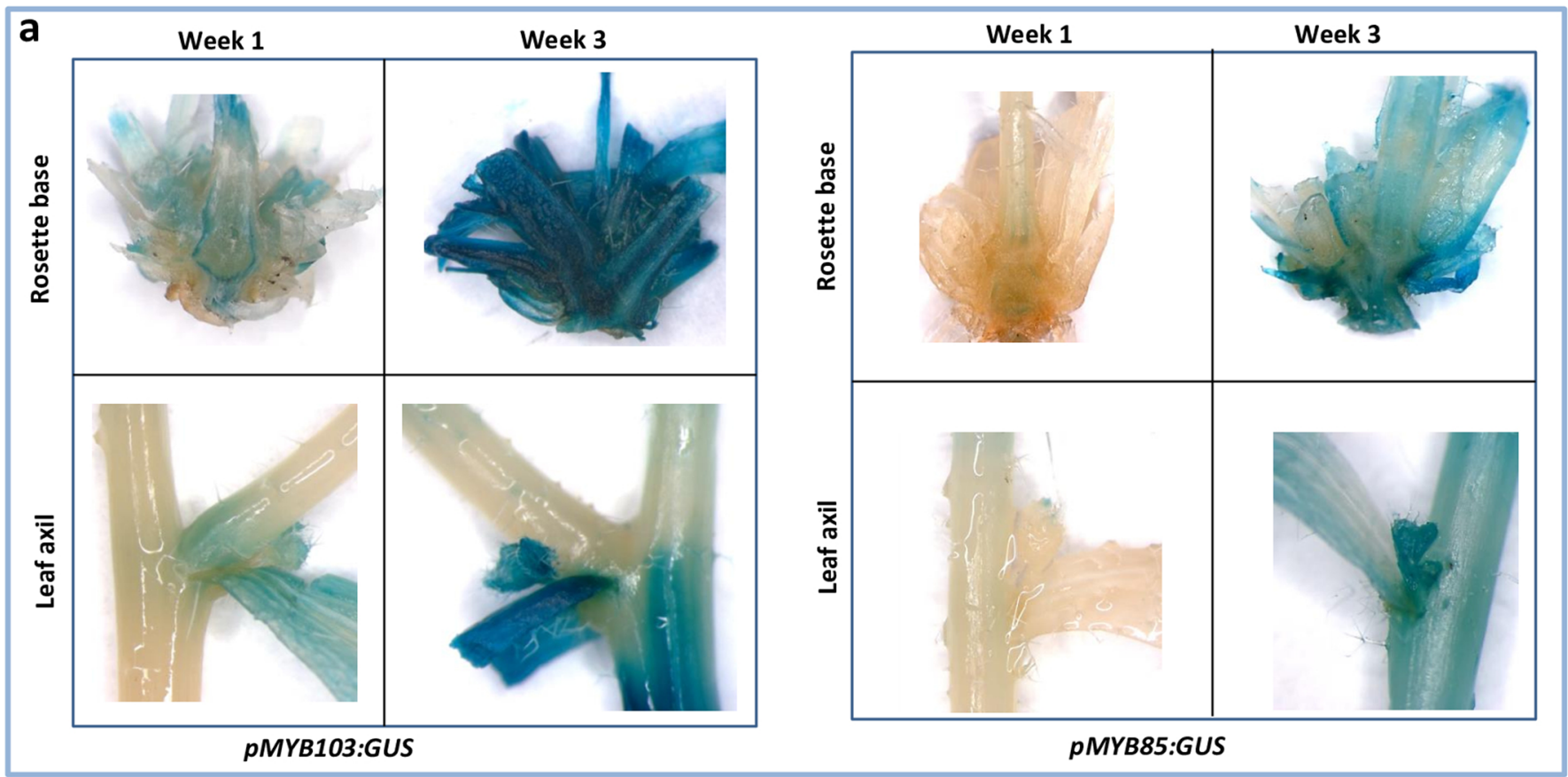

b

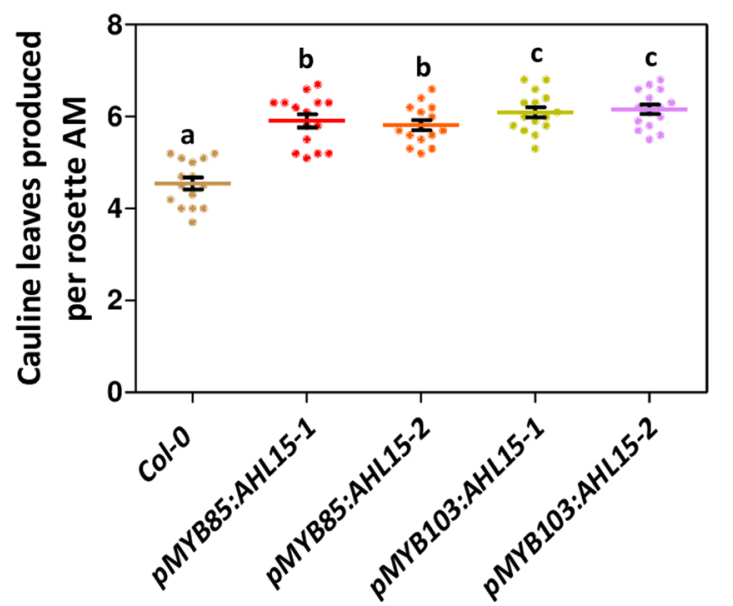

C

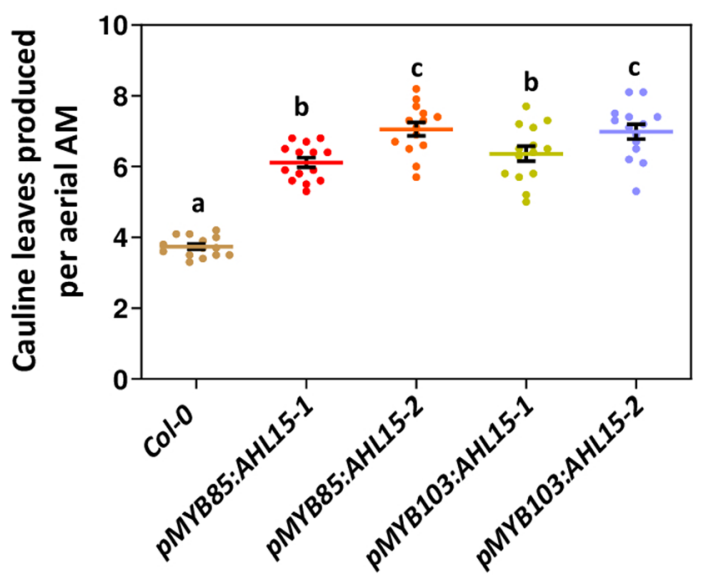

Extended Data Fig. 8 | AHL15 overexpression in the rosette base and leaf axils delays AM maturation in Arabidopsis. a. Expression of pMYB58:GUS and pMYB103:GUS reporters in the rosette base (top) or leaf axils (bottom) of Arabidopsis plants respectively one or three weeks after flowering, as monitored by histochemical GUS staining. Similar results were obtained in two independent experiments. $\mathbf{b}, \mathbf{c}$. The number of cauline leaves produced by rosette AMs (b) or aerial AMs (c) of 6-week-old (b) or 7-week-old (c) wild-type, pMYB85:AHL15 or pMYB103:AHL15 plants grown in LD conditions. Dots in b and c indicate number of cauline leaves produced per AM per plant ( $n=15$ biologically independent plants), horizontal lines indicate the mean, and error bars the s.e.m. Letters $(a, b, c)$ indicate statistically significant differences $(P<0.01)$, as determined by a one-way ANOVA with a Tukey's HSD post hoc test. The $P$ values can be found in Supplementary Tables 16 and 17. 

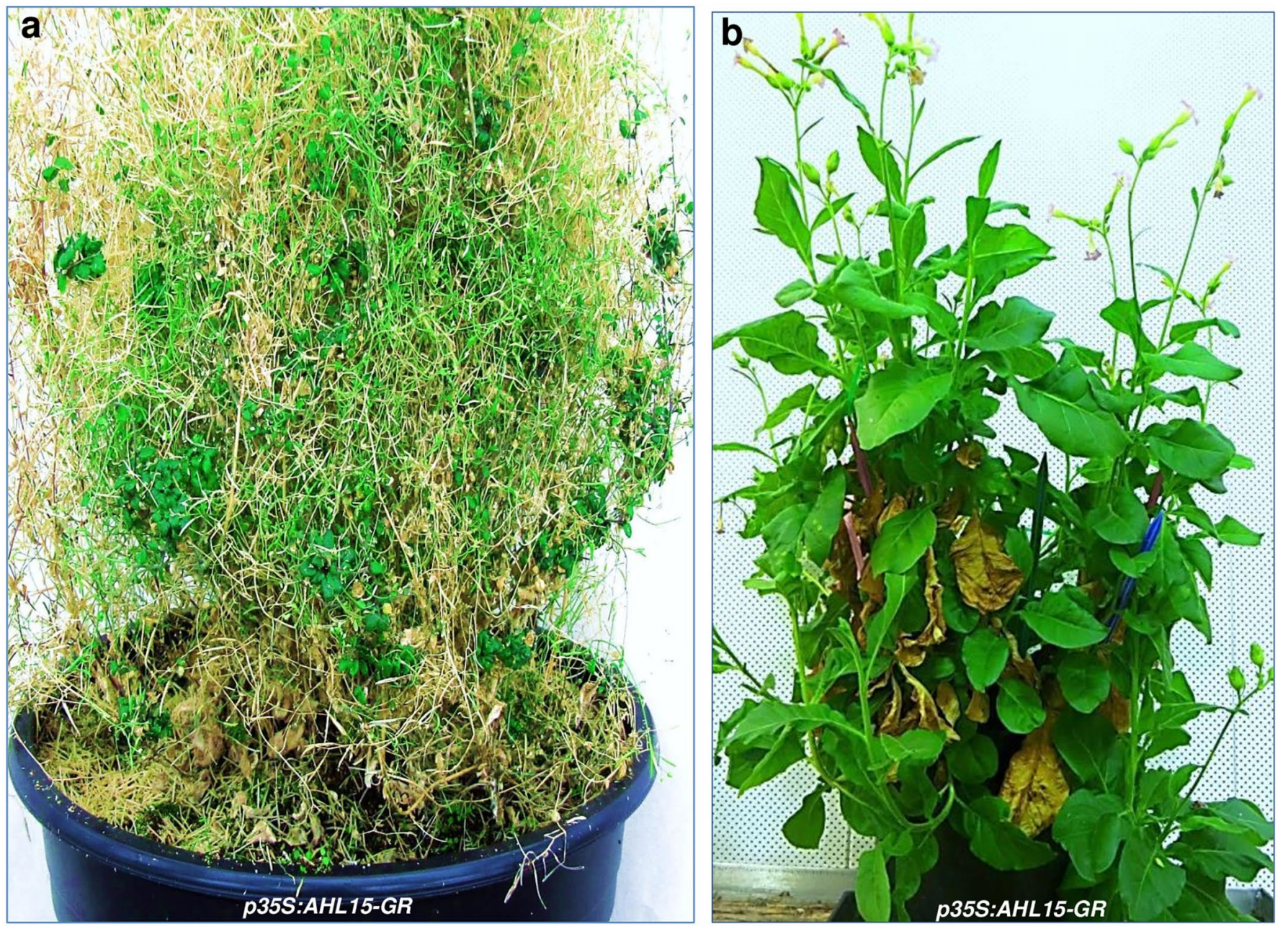

Extended Data Fig. 9| AHL15 overexpression promotes longevity in Arabidopsis and tobacco. a. Renewed vegetative growth on aerial branches of a 5-month-old Arabidopsis 35S:AHL15-GR plant, 4 weeks after spraying with $20 \mu$ M DEX. Similar results were obtained in three independent experiments. b. Efficient production of leaves and inflorescences in a 3-year-old 35S::AHL15-GR tobacco plant, 3 weeks after treatment with $30 \mu \mathrm{M}$ DEX, following 6 previous cycles of DEX-induced seed production. Similar results were obtained in two independent experiments. Plants in $\mathbf{a}$ and $\mathbf{b}$ were grown in LD conditions. 


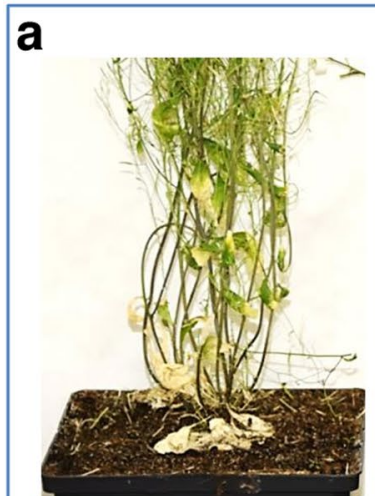

A. thaliana

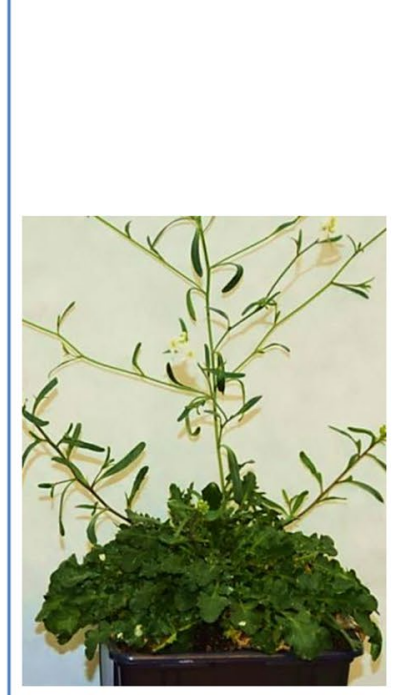

A. lyrata b

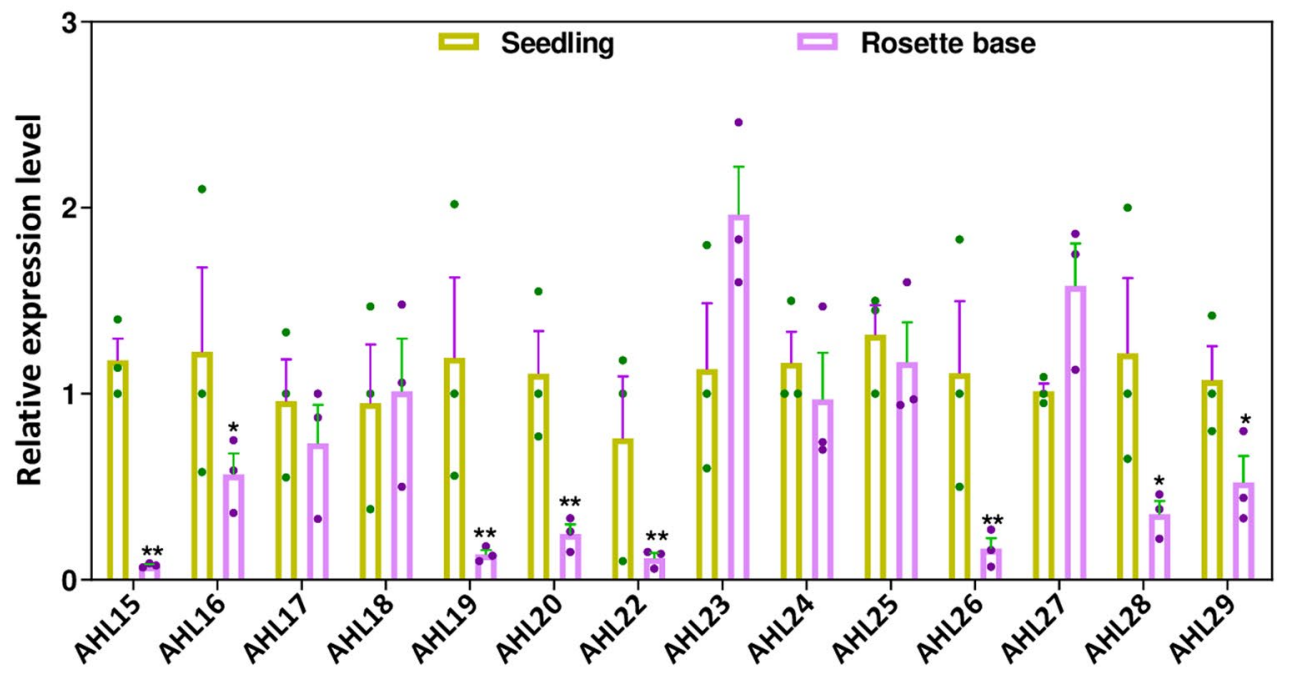

C

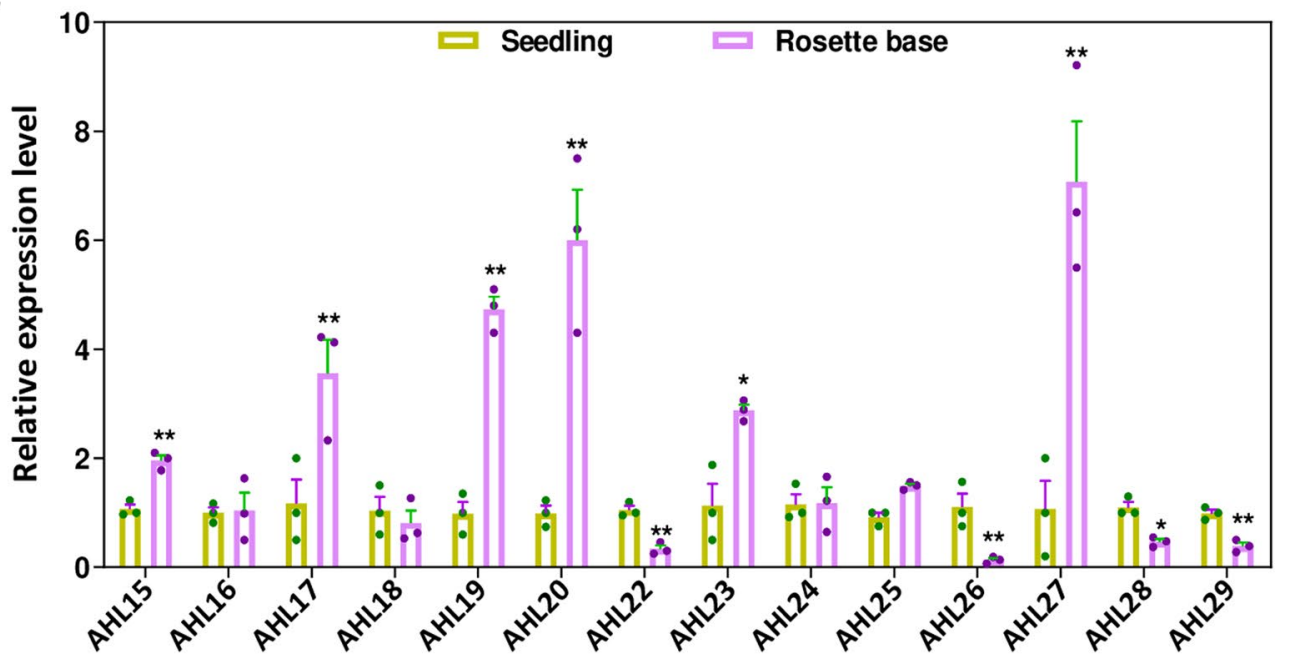

Extended Data Fig. 10 | Expression of clade-A AHL genes in seedlings or in the rosette base of flowering Arabidopsis or A. lyrata plants. a. Shoot phenotype of a 3-month-old Arabidopsis (upper panel) or a 4-month-old A. lyrata (lower panel) plant grown in LD conditions. Similar results were obtained in two independent experiments. b, c. qPCR analysis of the expression of clade-A AHL genes in 2-week-old seedlings or in the rosette base of 2-monthold flowering plants of $A$. thaliana (b) or $A$. lyrata (c). Dots in $\mathbf{b}$ and $\mathbf{c}$ indicate relative expression levels per experiment ( $n=3$ biologically independent replicates), bars indicate the mean, and error bars indicate the s.e.m. Asterisks indicate significant differences from mock-treated plants ${ }^{\star} p<0.05$, $\left.{ }^{\star \star} \mathrm{p}<0.01,{ }^{\star \star \star} \mathrm{p}<0.001\right)$, as determined by a two-sided Student's $t$-test. 


\section{Reporting Summary}

Nature Research wishes to improve the reproducibility of the work that we publish. This form provides structure for consistency and transparency in reporting. For further information on Nature Research policies, see Authors \& Referees and the Editorial Policy Checklist.

\section{Statistics}

For all statistical analyses, confirm that the following items are present in the figure legend, table legend, main text, or Methods section.

n/a Confirmed

$\square \searrow$ The exact sample size $(n)$ for each experimental group/condition, given as a discrete number and unit of measurement

$\square \bigotimes$ A statement on whether measurements were taken from distinct samples or whether the same sample was measured repeatedly

$\square$ The statistical test(s) used AND whether they are one- or two-sided

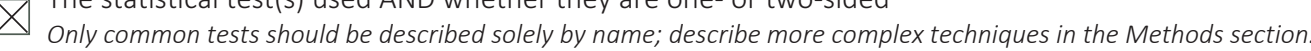

$\bigotimes \square$ A description of all covariates tested

$\bigotimes \square$ A description of any assumptions or corrections, such as tests of normality and adjustment for multiple comparisons

A full description of the statistical parameters including central tendency (e.g. means) or other basic estimates (e.g. regression coefficient) AND variation (e.g. standard deviation) or associated estimates of uncertainty (e.g. confidence intervals)

X $\square$ For null hypothesis testing, the test statistic (e.g. $F, t, r$ ) with confidence intervals, effect sizes, degrees of freedom and $P$ value noted

X Give P values as exact values whenever suitable.

$\bigotimes \square$ For Bayesian analysis, information on the choice of priors and Markov chain Monte Carlo settings

$\bigotimes \square$ For hierarchical and complex designs, identification of the appropriate level for tests and full reporting of outcomes

$\bigotimes \square$ Estimates of effect sizes (e.g. Cohen's $d$, Pearson's $r$ ), indicating how they were calculated

Our web collection on statistics for biologists contains articles on many of the points above.

\section{Software and code}

Policy information about availability of computer code

Data collection Biomart v0.9, BlastP 2.10,

Data analysis MAFFT7.4, trimAL1.2, HMMER3.1b1, PhyML3.0, MrBayes3.2.7, NOTUNG2.9, PhyloT, R packages APE5.3 and GEIGER2.0

For manuscripts utilizing custom algorithms or software that are central to the research but not yet described in published literature, software must be made available to editors/reviewers. We strongly encourage code deposition in a community repository (e.g. GitHub). See the Nature Research guidelines for submitting code \& software for further information.

\section{Data}

Policy information about availability of data

All manuscripts must include a data availability statement. This statement should provide the following information, where applicable:

- Accession codes, unique identifiers, or web links for publicly available datasets

- A list of figures that have associated raw data

- A description of any restrictions on data availability

All data generated or analysed during this study are included in this published article (and its supplementary information files).

\section{Field-specific reporting}

Please select the one below that is the best fit for your research. If you are not sure, read the appropriate sections before making your selection.
\ Life sciences
$\square$ Behavioural \& social sciences
Ecological, evolutionary \& environmental sciences

For a reference copy of the document with all sections, see nature.com/documents/nr-reporting-summary-flat.pdf 


\section{Life sciences study design}

All studies must disclose on these points even when the disclosure is negative.

Sample size

No statistical method was used to determine sample size. Sample size was chosen based on what is common in the field

Data exclusions

No data have been excluded from our analysis

Replication

All described experiments were repeated at least once, and appeared reproducible.

Randomization

For plant phenotyping and morphometry, progeny of a genetically homozygous plant parent were randomly selected.

Blinding

Blinding was not relevant in our study, since the phenotypic differences between the different lines were sufficiently large.

\section{Reporting for specific materials, systems and methods}

We require information from authors about some types of materials, experimental systems and methods used in many studies. Here, indicate whether each material, system or method listed is relevant to your study. If you are not sure if a list item applies to your research, read the appropriate section before selecting a response.

Materials \& experimental systems Methods

$\mathrm{n} / \mathrm{a}$ Involved in the study

$\square$ Antibodies, Anti-GFP, see ref. 45/46 $\mathrm{n} / \mathrm{a}$ Involved in the study

Х Eukaryotic cell lines

Х Palaeontology

$\triangle \square$ ChIP-seq

X $\square$ Animals and other organisms

$\bigotimes \square$ Flow cytometry

$\square$ Human research participants

$\bigotimes \square$ Clinical data 